\title{
Combined transmit beamforming and channel-aware scheduling for interference mitigation in femtocells
}

\author{
Alexis A Dowhuszko ${ }^{1 *}$, Mika Husso ${ }^{1}$ and Jyri Hämäläinen 1,2
}

\section{*Correspondence:}

alexis.dowhuszko@aalto.fi

${ }^{1}$ Department of Communications and Networking, Aalto University,

P.O. Box 13000, Fl-00076 Aalto,

Finland

Full list of author information is

available at the end of the article

\section{Introduction}

Eventhough the concept of the home base station (HBS) was introduced as early as 1996 $[1,2]$ and Alcatel was planning to commercialize it in 2000 [3], it took until around 20072008 for the research and concurrent business activities to gain real momentum. The femtocell term, then, was adopted around 2005 for a standalone, self-configuring low-power base station [4]. Currently, femtocells constitute an important study topic with a significant body of accumulated research, receiving wide industry support at the same time.

Adding the femtocell component into the existing macrocellular networks raises a number of technical challenges related to, e.g., timing and synchronization, spectrum allocation and interference management [5]. Eventhough mobile operators may in principle allocate a separate carrier for femtocell operations (eliminating thereby the cross-layer interference problem), constraints related to spectrum availability and costs often make this approach unattractive or unfeasible. However, even in presence of a dedicated band for femto-femto operation, interference still remains as a serious problem: the uncoordinated nature of femtocell deployments generates a great variability in the quality of service (QoS) that the users perceive in the network.

The problems of both, cross-layer and co-layer interference in an heterogenous network that combines multiple layers have been widely addressed in academic research. While the first publications [5-7] concentrated on characterizing and quantifying the problem, more recent studies have typically focused on methods for interference coordination and mitigation utilizing different approaches to tackle the problem. In, e.g., [8,9], the authors 
proposed the use of dynamic frequency re-use, whereas in [10,11] power control was utilized for downlink and uplink cross-layer interference mitigation purposes, respectively. In addition, contributions of [12-14] explored the use of transmit beamforming (TBF) methods for interference mitigation that rely on explicit channel state information (CSI) reports in the reverse channel (i.e., for FDD air interfaces), while [15] analyzed the use of a busy burst protocol to coordinate the selection of TBF vectors at neighboring cells when channel reciprocity holds (i.e., for TDD air interfaces). In [16], a scheduling method was presented for reducing macro-femto interference, and in $[17,18]$ beamforming and scheduling were combined for inter-femtocell interference mitigation purposes. It is important to highlight that, within LTE-Advanced nomenclature, 3GPP standardization has given the name enhanced intercell interference coordination (eICIC) to categorize those techniques that are designed to mitigate interference in an heterogeneous network scenario. A good summary of them can be found, e.g., in [19] and references therein.

In this article, we present a low-rate feedback method for co-layer interference mitigation and cell balancing in the downlink of a closed-access femtocell. Using a combination of practical TBF methods and simple channel-aware inter-cell scheduling algorithms, we show that resources (i.e., degrees of freedom in the spatial and temporal domains) can be transferred from a cell operating in high SINR regime to a cell that is operating with low SINR. In other words, the proposed method is able to effectively balance the downlink data rate that is achieved at each cell, requiring only the presence of a low-rate uplink control channel between the interfering femto access point (FAP) and the victim femto user equipment (FUE). We note that a similar method, known as enhanced codebook-based transmission, was presented in [20] and references therein as one potential solution for performance enhancements in single- and multi-cell LTE-Advanced networks. Nevertheless, the main difference with our proposal lies in the fact that in a FAP, the number of associated FUEs is expected to be much lower than the number of potential candidates to be served in a macro base station. This limitation precludes the possibility of finding a best companion (for user pairing) and worst companion (for interference coordination) to be served simultaneously at each transmission time instant.

The rest of the article is structured as follows. Section 'System model' presents the general system and the adopted assumptions, the applied TBF schemes, and the channelaware scheduling algorithms that are analyzed. Section 'Theoretical analysis' derives the theoretical formulas that are required to evaluate the performance of our proposed method, whereas Section 'Performance analysis' shows the corresponding results that were obtained (both, theoretical, and numerical ones). Finally, Section 'Conclusions and discussion' concludes the article and enumerates open issues that are suitable for future research study.

\section{System model}

This section presents the general system model and the assumptions that are adopted for the closed-access femtocellular system. The TBF schemes and the channel-aware scheduling algorithms are also introduced in this section.

\section{Interference scenario and adopted assumptions}

The analyzed system consists of two closed-access FAPs that are equipped with $M$ transmit antennas, and two single-antenna FUEs. Each FUE communicates to its serving FAP 
the TBF vector $\widehat{w}$ that maximizes its received SINR. In addition, when experiencing high interference originated in a neighboring femtocell, we assume that each (victim) FUE has the chance to inform the TBF vector $\breve{w}$ that would minimize the received interference power when applied in transmission at the corresponding (interfering) FAP. Finally, each FUE can request the interfering FAP to temporarily stop transmission by issuing a silence request (SR) message. In order to convey this interference mitigation related feedback, we consider the existence of an additional low-rate reverse control channel between each victim FUE and the neighboring FAP that is responsible of the largest interference contribution. For an illustrative purpose, the general system model when $M=2$ is presented in Figure 1.

The implementation of our interference coordination approach demands the exchange of control information between victim FUE and interfering FAP. In our system model, we consider that this signaling exchange is done over the air in a single-hop fashion, relaxing the requirement of having a backhaul femto gateway (FGW) connection that supports real time control message exchange using a two-hop approach (i.e., relaying the control information from serving to interfering FAP). Note that actually, 3GPP Rel. 10 supports an X2-like interface between FAPs. However, the information exchange over X2 is time constrained due to delay issues, especially when dealing with FAPs that belong to different closed subscriber groups (CSGs). Although it will take some years until Rel. 10 products become widely available in the market, the main limitation for using control messages over X2 is that the quality of this interface is not expected to improve considerably in the future (especially for residential customers that use xDSL connections as backhaul). Thus, standardizing effective over the air control mechanisms is important, to be able to implement interference coordination schemes within femtocell environments in a simple way.

Note that in our system scenario, we consider that both femtocells are located close to each other. Therefore, if we consider that a given victim FUE is experiencing strong interference from a neighboring FAP (in the direct-link), we can also assume that the signal strength in the reverse channel (i.e., from the victim FUE to the interfering FAP) will be also good enough to guarantee a proper exchange of control messages for interference coordination. Nevertheless, it is important to notice that all the analysis that is presented in this article does not preclude the use of a two-hop signaling scheme (e.g., (1) FUE $1 \rightarrow$

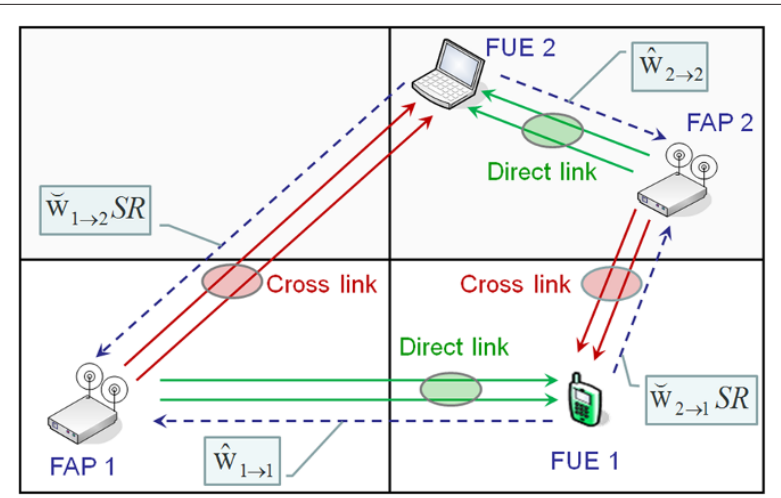

Figure 1 Illustration of an interference scenario between two closed-access femtocells. Channel between FAP 2 and its FUE 2 is good, while FUE 1 connected to FAP 1 may face heavy interference from FAP 2. 
FAP 1, (2) FAP $1 \rightarrow$ FAP 2) whenever a reliable backhaul interface between neighboring FAPs is available. Details of the feedback channel and the interference mitigation methods will be discussed in Sections 'Transmit beamforming' and 'Channel-aware scheduling of silence periods'.

\section{Adopted assumptions}

We have adopted the following baseline assumptions regarding the general framework:

(A1) We analyze a two-cell scenario where an adjacent FAP creates downlink co-channel interference to the FUE of the reference cell. Transmission power in the FAPs is constant and handover between cells is not possible. In other words, CSG configuration is applied, but the FUE can establish a control connection to both, the serving and the interfering FAP unit.

(A2) There are $M$ transmit antennas in both FAPs, and a single receive antenna in each FUE. The terminal can estimate signals from the different antennas of both, the serving FAP and the interfering FAP. Throughout this article, channel estimation is assumed to be perfect at the receiver side. The terminal can send a quantized feedback message to both femtocellular transceivers, including information for the antenna weight selection and SR messages. The impact of feedback delay and feedback errors is ignored.

(A3) Channel gains related to the different antennas of a given FAP are considered as independent and identically distributed (i.i.d.) complex zero-mean Gaussian random variables (RVs), while the mean received power is different for different FAPs. Fast fading corresponding to different FAPs is assumed uncorrelated.

\section{Transmit beamforming}

To implement TBF in a FDD system, a base station makes use of quantized CSI, encoded into a feedback message that is sent from the mobile terminal through a reverse control channel. In LTE, e.g., there is a physical control channel that carries feedback messages and may be used to convey CSI from the mobile terminal to the base station $[21,22]$. In our study, feedback words include short-term CSI, which is assumed to be available at the transmitter without errors or latency. For the analysis in the presence of feedback errors refer to, e.g., [23].

In an interference-free case, the received signal at the mobile terminal $k$ is given by

$$
r_{k}=\left(\mathbf{h}_{k \rightarrow k} \cdot \mathbf{w}_{k}\right) s_{k}+n_{k}=\left(\sum_{m=1}^{M} h_{k \rightarrow k}^{(m)} w_{k}^{(m)}\right) s_{k}+n_{k},
$$

where $M$ is the number of transmit antennas, $s_{k}$ is the transmitted complex baseband symbol, $\mathbf{h}_{k \rightarrow k}=\left[h_{k \rightarrow k}^{(1)} \ldots h_{k \rightarrow k}^{(M)}\right]$ is the channel gain vector between mobile terminal $k$ and each transmit antenna $m$ of its serving base station (composed of zero-mean complex Gaussian coefficients), $\mathbf{w}_{k}=\left[w_{k}^{(1)} \ldots w_{k}^{(M)}\right]$ is the complex beamforming vector that the serving base station applies, and $n_{k}$ refers to zero-mean complex additive white Gaussian noise with power $P_{N}$. Note that in our signal model, term $n_{k}$ reflects not only the effect of the thermal noise of the system, but also the effect of all those interference sources whose contribution cannot be estimated independently in reception (e.g., co-channel and 
adjacent-channel interference originated in both, macro- and femto-layers of the heterogeneous network). Since $n_{k}$ groups a large number of non-separable sources of interference, it is treated as white Gaussian noise when performing decoding at the receiver side. Complex transmit weights are normalized such that $\left\|\mathbf{w}_{k}\right\|^{2}=\sum_{m=1}^{M}\left|w_{k}^{(m)}\right|^{2}=1$. We note that signal model (1) assumes a frequency flat fading channel. For a frequency selective fading channel, the same signal model can still be used if the total bandwidth of the system $B$ is divided into sub-bands that have an approximately frequency flat response, and TBF feedback is provided for each sub-band separately.

We assume that there are two FAPs (denoted by indices $k=1,2$ ), each serving one FUE with equal transmit power $P_{\mathrm{Tx}}=\mathbb{E}\left\{\left|s_{k}\right|^{2}\right\}$. Then, the achievable rates for bandwidth $B$ are given by

$$
\begin{aligned}
& R_{1}\left(\mathbf{w}_{1}, \mathbf{w}_{2}\right)=B \log _{2}\left(1+\frac{P_{\mathrm{Tx}}\left|\mathbf{h}_{1 \rightarrow 1} \cdot \mathbf{w}_{1}\right|^{2}}{P_{\mathrm{Tx}}\left|\mathbf{h}_{2 \rightarrow 1} \cdot \mathbf{w}_{2}\right|^{2}+P_{N}}\right), \\
& R_{2}\left(\mathbf{w}_{2}, \mathbf{w}_{1}\right)=B \log _{2}\left(1+\frac{P_{\mathrm{Tx}}\left|\mathbf{h}_{2 \rightarrow 2} \cdot \mathbf{w}_{2}\right|^{2}}{P_{\mathrm{Tx}}\left|\mathbf{h}_{1 \rightarrow 2} \cdot \mathbf{w}_{1}\right|^{2}+P_{N}}\right),
\end{aligned}
$$

where $\mathbf{h}_{k \rightarrow l}$ is the channel gain vector from FAP $k$ to FUE $l$, while $\mathbf{w}_{k}$ is the corresponding beamforming vector that the FAP $k$ applies in transmission. Note that in (2) and (3), the dominant interference originated in the neighboring femtocell is treated as an extra component of additive white Gaussian noise, simplifying the implementation of the decoder at the receiver side (i.e., no advanced signal decoding and/or interference cancelation techniques are considered for this purpose). Now, related to the selection of $\mathbf{w}_{1}$ and $\mathbf{w}_{2}$, we introduce two basic beamforming strategies.

\section{Egoistic and altruistic beamforming}

In egoistic beamforming, each FAP selects for transmission the beamforming vector $\hat{\mathbf{w}}$ that maximizes the rate towards its own FUE. Correspondingly, a strategy in which the FAP applies the beamforming vector $\check{\mathbf{w}}$ that minimizes interference to the FUE in the neighboring femtocell will be referred to as altruistic beamforming.

When both FAPs implement egoistic beamforming, the achievable rates for bandwidth $B$ are given by

$$
\begin{aligned}
& R_{1}^{\text {(ego/ego) }}\left(\hat{\mathbf{w}}_{1}, \hat{\mathbf{w}}_{2}\right)=B \log _{2}\left(1+\frac{P_{\mathrm{Tx}}\left|\mathbf{h}_{1 \rightarrow 1} \cdot \hat{\mathbf{w}}_{1}\right|^{2}}{P_{\mathrm{Tx}}\left|\mathbf{h}_{2 \rightarrow 1} \cdot \hat{\mathbf{w}}_{2}\right|^{2}+P_{N}}\right), \\
& R_{2}^{\text {(ego/ego) }}\left(\hat{\mathbf{w}}_{2}, \hat{\mathbf{w}}_{1}\right)=B \log _{2}\left(1+\frac{P_{\mathrm{Tx}}\left|\mathbf{h}_{2 \rightarrow 2} \cdot \hat{\mathbf{w}}_{2}\right|^{2}}{P_{\mathrm{Tx}}\left|\mathbf{h}_{1 \rightarrow 2} \cdot \hat{\mathbf{w}}_{1}\right|^{2}+P_{N}}\right) .
\end{aligned}
$$

On the other hand, when FAP 1 implements egoistic beamforming (i.e., applies $\hat{\mathbf{w}}_{1}$ ) and FAP 2 altruistic beamforming (i.e., applies $\check{\mathbf{w}}_{2}$ ), the equivalent equations for the achievable rates become

$$
\begin{aligned}
& R_{1}^{\text {(ego/alt) }}\left(\hat{\mathbf{w}}_{1}, \check{\mathbf{w}}_{2}\right)=B \log _{2}\left(1+\frac{P_{\mathrm{Tx}}\left|\mathbf{h}_{1 \rightarrow 1} \cdot \hat{\mathbf{w}}_{1}\right|^{2}}{P_{\mathrm{Tx}}\left|\mathbf{h}_{2 \rightarrow 1} \cdot \check{\mathbf{w}}_{2}\right|^{2}+P_{N}}\right), \\
& R_{2}^{\text {(alt/ego) }}\left(\check{\mathbf{w}}_{2}, \hat{\mathbf{w}}_{1}\right)=B \log _{2}\left(1+\frac{P_{\mathrm{Tx}}\left|\mathbf{h}_{2 \rightarrow 2} \cdot \check{\mathbf{w}}_{2}\right|^{2}}{P_{\mathrm{Tx}}\left|\mathbf{h}_{1 \rightarrow 2} \cdot \hat{\mathbf{w}}_{1}\right|^{2}+P_{N}}\right) .
\end{aligned}
$$

As expected, the implementation of altruistic beamforming at FAP 2 makes FUE 2 to lose the beamforming gain (because the selection of $\mathbf{w}_{2}$ becomes independent of $\mathbf{h}_{2 \rightarrow 2}$ ). It is important to highlight that, most part of the analysis presented in this article deals with 
the latter case (i.e., combination of altruistic and egoistic beamforming in a two-femtocell scenario).

In order to link the study to practical systems, we have employed a simple TBF method (known as closed-loop mode 1) that resembles the one applied in HSDPA in presence of two transmit antennas [24]. A similar method has also been adopted in LTE and, although our method is not exactly the same, the performance difference between HSDPA closedloop method and its LTE counterpart is negligible. In HSDPA, the mobile user defines the 1-bit (phasing) message that is reported to the serving base station at each time instant. The base station thus maintains the phase at the first antenna, and then adjusts the phase of the second antenna based on the sliding averaging over two consecutive feedback commands, see [25] for more details. Note that if the speed of the mobile user is low (or equivalently, the coherence time of the channel is large compared to the signaling delay), closed-loop mode 1 is equivalent to have error-free QPSK phasing information at the second antenna. In other words, we can assume that transmit beamforming weights are not affected by the signaling delay, taking the form $\frac{1}{\sqrt{2}}\left[1 e^{j(2 n+1) \frac{\pi}{4}}\right]$, for $n=0,1,2,3$.

In this article, we consider a generalized mode 1 with $N$ phasing bits. Instead of standard QPSK phasing (i.e., 2-bit mode 1), we analyze the equivalent mode 1 versions with $N=0,1,2$ (phasing bits/antenna), to illustrate the gain that additional resolution in the phasing information provides. The full channel phase information case (i.e., $N \rightarrow \infty$ ) is also considered, to determine the performance upper bound for the scheme. In addition, we shall discuss the problem of feedback bit allocation between scheduling and TBF. Although $N$-bit phasing is not an optimal closed-loop method, it admits closed-form formulations for performance measures (e.g., the outage probability). As a consequence, it allows to obtain a better insight to the system performance, than the one that would be achieved through a pure computer simulation study.

\section{Channel-aware scheduling of silence periods}

In the proposed method, the scheduling decisions of silence periods (SPs) can be based on the strength and timing of the transmissions from the dominant interfering femtocell. Thus, whenever there are free time resources available in the interfering FAP, they can be used to provide periods of silence that improve the achievable rates of victim FUEs in adjacent cells. Scheduling of such interference free periods is implemented as follows:

- Victim FUE transmits a SR message to the interfering FAP whenever it experiences high levels of interference. To keep the analysis tractable, we consider that interfering FAP always grants a SP when requested.

- Victim FUE is able to select the best moment to send SR easily, if interfering FAP informs the availability of interference-free resources in time domain (e.g., percentage of time frames in which transmission could be stopped under request). We consider that victim FUE is able to monitor these messages, e.g., listening to the broadcast channel of the adjacent femtocell.

- If SRs are not received at interfering FAP, it does not need to issue a SP at that time frame.

Different scheduling algorithms can be constructed to determine the best time instant in which a victim FUE should issue a SR to its interfering FAP. In its simpler form, scheduling of SP can follow two options: 
1. Cross-link scheduling criterion, or

2. Direct-link scheduling criterion.

Cross-link scheduling criterion can be expressed as

$$
\frac{\left\|\mathbf{h}_{k \rightarrow l}\right\|^{2}}{E\left\{|| \mathbf{h}_{k \rightarrow l} \|^{2}\right\}}>\hat{\gamma}_{\mathrm{th}}^{(c)} \quad k \neq l,
$$

where $\hat{\gamma}_{\text {th }}^{(c)}$ is a threshold that can be selected based on the service requirements. As secondary option, scheduling decisions can be made using own signal strength. Then, the victim FUE can request a SP from the interfering FAP when the communication link towards its serving FAP is strong, i.e.,

$$
\frac{\left\|\mathbf{h}_{k \rightarrow k}\right\|^{2}}{E\left\{\left\|\mathbf{h}_{k \rightarrow k}\right\|^{2}\right\}}>\hat{\gamma}_{\mathrm{th}}^{(d)} .
$$

Finally, if we desire to maximize cell edge performance (e.g., the 10th percentile of a cumulative distribution function), then we may send a SR when we experience a weak channel in our direct-link, i.e.,

$$
\frac{\left\|\mathbf{h}_{k \rightarrow k}\right\|^{2}}{E\left\{\left\|\mathbf{h}_{k \rightarrow k}\right\|^{2}\right\}}<\check{\gamma}_{\mathrm{th}}^{(d)} .
$$

Eventhough the different SP scheduling criteria (8)-(10) are presented as functions of the normalized received powers in both, cross-link and direct-link, SPs can also be triggered as functions of the received SNR in both links. The latter case is simpler to implement in practice, since the SNR value of both links can be easily estimated (at FUEs) measuring the quality of the pilot signals that FAPs broadcast in downlink. Note that in this case, the precise value that the scheduling threshold should take will be also affected by the background noise power that the victim FUE experiences in reception (i.e., $P_{N}$ ).

In this article, we mainly aim at improving low SINR performance. Therefore, we shall focus on $\hat{\gamma}_{\text {th }}^{(c)}$ and $\check{\gamma}_{\text {th }}^{(d)}$ as the scheduling thresholds. The analysis that forms the basis for the performance evaluation is carried out in the following section.

\section{Theoretical analysis}

The analysis in this section is done using the cumulative distribution function (CDF) of the received SINR as the performance measure. Later on, we extend the analysis to the CDF of the throughput of the FUEs. Note that part of these results has been presented in [26], where closed-form expressions for the received SINR of TBF methods were derived, without scheduling SPs according to channel conditions.

\section{Cumulative distribution function for SINR of mode 1}

Keeping in mind the computation of the received SINR, we first consider the RV

$$
Z=\frac{X}{1+Y}
$$


assuming that RVs $X$ and $Y$ are independent. In this situation, the CDF of $Z$ is of the form

$$
F_{Z}(z)=\int_{1}^{+\infty} F_{X}(z t) f_{Y}(t-1) d t=\int_{0}^{+\infty} F_{X}[z(t+1)] f_{Y}(t) d t,
$$

where $f_{Y}(y)$ is the probability density function (PDF) of $Y$, while $F_{X}(x)$ represents the CDF of $X[27]$.

To derive the stochastic behavior of

$$
\Upsilon_{(1)}=\frac{\bar{\gamma}_{1 \rightarrow 1}\left|\mathbf{h}_{1 \rightarrow 1} \cdot \widehat{\mathbf{w}}_{1}\right|^{2}}{1+\bar{\gamma}_{2 \rightarrow 1}\left|\mathbf{h}_{2 \rightarrow 1} \cdot \check{\mathbf{w}}_{2}\right|^{2}},
$$

where

$$
\bar{\gamma}_{k \rightarrow l}=\frac{P_{\mathrm{Tx}}}{\bar{L}_{k \rightarrow l} P_{N}} \quad \forall k, l
$$

is the mean received SNR for the link between FAP $k$ and FUE $l$ (assumed constant during the whole duration of the communication), while $\bar{L}_{k \rightarrow l}$ represents the corresponding distance dependent path loss attenuation (i.e., long-term component of the fading channel), we need to compute formula (12) assuming

$$
X=\bar{\gamma}_{1 \rightarrow 1}\left|\mathbf{h}_{1 \rightarrow 1} \cdot \widehat{\mathbf{w}}_{1}\right|^{2}, \quad Y=\bar{\gamma}_{2 \rightarrow 1}\left|\mathbf{h}_{2 \rightarrow 1} \cdot \check{\mathbf{w}}_{2}\right|^{2} .
$$

Unfortunately, exact distribution functions for $X$ and $Y$ are difficult to obtain in this situation. So, we make use of the chi-square $\left(\chi^{2}\right)$ distribution approximations presented in [28]:

$$
\begin{array}{lll}
F_{X}(x)=1-\left(1+\frac{2 x}{\mathcal{G} \bar{\gamma}_{1 \rightarrow 1}}\right) e^{-\frac{2 x}{\mathcal{G} \bar{\gamma}_{1 \rightarrow 1}}} & x \geq 0, & \mathcal{G}=\mathbb{E}\left\{\left|\mathbf{h}_{1 \rightarrow 1} \cdot \widehat{\mathbf{w}}_{1}\right|^{2}\right\}, \\
f_{Y}(y)=\frac{1}{g \bar{\gamma}_{2 \rightarrow 1}} e^{-\frac{y}{g \bar{\gamma}_{2 \rightarrow 1}}} & y \geq 0, & g=\mathbb{E}\left\{\left|\mathbf{h}_{2 \rightarrow 1} \cdot \check{\mathbf{w}}_{2}\right|^{2}\right\} .
\end{array}
$$

Expectations $\mathcal{G}$ and $g$ are known as the beamforming gains (or coherent combining gains) of the TBF scheme, and can be interpreted as scaling factors that reflect the impact of incomplete CSI in the system. In case of mode 1, the beamforming gains admit closedform expressions [29]

$$
\mathcal{G}=1+\frac{\pi}{4} a_{N}, \quad g=1-\frac{\pi}{4} a_{N}, \quad a_{N}=\frac{2^{N}}{\pi} \sin \left(\frac{\pi}{2^{N}}\right) .
$$

The use of a $\chi^{2}$ distributions with 4 (2) degrees of freedom has been previously proposed in [30], as a simple way to approximate the stochastic behavior of the desired signal energy (mutual interference) that a user experiences in reception, when linear beamforming is applied in transmission. In [30], the authors used this approximation to evaluate the effect of limited CSI in a MIMO broadcast channel, when spatial multiplexing is implemented in transmission using similar codebook structures (e.g., closed-loop mode 1). The justification for such an approach can be found in [29], and is based on the fact that the Nakagami distribution shows good fitting when modeling the received SNR that various multi-antenna techniques provide. In practice, the degrees of freedom for the corresponding $\chi^{2}$ approximation depend on the value that the fading figure parameter takes (by definition, the fading figure of a Nakagami RV is the ratio of its squared mean value to 
its variance). Note that when this value is rounded to the closest even integer, its use enables to derive closed-form expressions which estimate different performance indicators with excellent fitting to simulated values. Results presented in [26], e.g., are based on this approach.

In order to simplify notation in the following equations, we define

$$
v_{1}=\frac{\bar{\gamma}_{1 \rightarrow 1}}{\bar{\gamma}_{2 \rightarrow 1}}, \quad v_{2}=\frac{\bar{\gamma}_{2 \rightarrow 2}}{\bar{\gamma}_{1 \rightarrow 2}} \text {. }
$$

Then, after some manipulations we obtain

$$
F_{\Upsilon_{(1)}}(z)=1-e^{-\frac{2 z}{\mathcal{G} \bar{\gamma}_{1 \rightarrow 1}}}\left[\frac{\frac{2 z v_{1} \mathcal{G}}{g}}{\left(\frac{v_{1} \mathcal{G}}{g}+2 z\right)^{2}}+\frac{\left(1+\frac{2 z}{\mathcal{G} \bar{\gamma}_{1 \rightarrow 1}}\right) \frac{\nu_{1} \mathcal{G}}{g}}{\frac{v_{1} \mathcal{G}}{g}+2 z}\right] .
$$

When the FAP 2 applies TBF for interference mitigation purposes in FUE 1, beamforming gain in the second femtocell vanishes since component channels sum up with random phases. In other words, the numerator of expression

$$
\Upsilon_{(2)}=\frac{\bar{\gamma}_{2 \rightarrow 2}\left|\mathbf{h}_{2 \rightarrow 2} \cdot \check{\mathbf{w}}_{2}\right|^{2}}{1+\bar{\gamma}_{1 \rightarrow 2}\left|\mathbf{h}_{1 \rightarrow 2} \cdot \widehat{\mathbf{w}}_{1}\right|^{2}}
$$

becomes exponentially distributed with mean value dictated by factor $\bar{\gamma}_{2 \rightarrow 2}$ (i.e., there is no beamforming gain). The same situation takes place in the second term of the denominator of (20), since the TBF vector that the FAP 1 applies does not take into account the actual values of channel gains in the corresponding cross-link. After some manipulations, it is possible to see that

$$
F_{\Upsilon_{(2)}}(z)=1-\frac{v_{2}}{v_{2}+z} e^{-\frac{z}{\gamma_{2} \rightarrow 2}}
$$

establishes the CDF expression for the SINR of the second femtocell user in this case.

It is important to note that when both FAPs carry out TBF independently (i.e., egoistic beamforming without considering interference mitigation issues), the SINR expressions become

$$
\Upsilon_{1}=\frac{\bar{\gamma}_{1 \rightarrow 1}\left|\mathbf{h}_{1 \rightarrow 1} \cdot \widehat{\mathbf{w}}_{1}\right|^{2}}{1+\bar{\gamma}_{2 \rightarrow 1}\left|\mathbf{h}_{2 \rightarrow 1} \cdot \widehat{\mathbf{w}}_{2}\right|^{2}}, \quad \Upsilon_{2}=\frac{\bar{\gamma}_{2 \rightarrow 2}\left|\mathbf{h}_{2 \rightarrow 2} \cdot \widehat{\mathbf{w}}_{2}\right|^{2}}{1+\bar{\gamma}_{1 \rightarrow 2}\left|\mathbf{h}_{1 \rightarrow 2} \cdot \widehat{\mathbf{w}}_{1}\right|^{2}}
$$

In this situation,

$$
F_{\Upsilon_{l}}(z)=1-e^{-\frac{2 z}{\mathcal{G} \bar{\gamma}_{l \rightarrow l}}}\left[\frac{2 z v_{l} \mathcal{G}}{\left(\nu_{l} \mathcal{G}+2 z\right)^{2}}+\frac{\left(1+\frac{2 z}{\mathcal{G} \bar{\gamma}_{l \rightarrow l}}\right) \nu_{l} \mathcal{G}}{\nu_{l} \mathcal{G}+2 z}\right] \quad l=1,2
$$

results when setting $g=1 \mathrm{in}(19)$. The latter expression reduces to

$$
F_{\Upsilon_{l}}(z)=1-e^{-\frac{2 z}{\mathcal{G} \bar{l}_{l \rightarrow l}}}\left(1+\frac{2 z}{\mathcal{G} \bar{\gamma}_{l \rightarrow l}}\right) \quad l=1,2
$$

when co-channel interference between both femtocells disappears (i.e., when $v_{l} \rightarrow \infty$ ). 


\section{Scheduling of silence periods based on cross-link signal strength}

In this section, we derive a closed-form expression to characterize the stochastic behavior of the received SINR when the inter-cell scheduling among femtocells is based on the strength of the interference link (i.e., the cross-link interference level). When combining TBF with a channel-aware scheduling of SPs according to (8), the CDF expression presented in (12) becomes

$$
F_{Z}(z)=\int_{0}^{\hat{\gamma}_{\mathrm{th}}} F_{X}[z(t+1)] f_{Y}(t) d t+F_{X}(z) \operatorname{Pr}\left\{y>\hat{\gamma}_{\mathrm{th}}\right\} \quad z \geq 0,
$$

where different probabilistic models are used to approximate the stochastic behaviors of RVs $X$ and $Y$, according to the number of phase bits $N$ that are used in the quantization set of the TBF scheme.

\section{Single-antenna system (no channel phase information)}

When the TBF scheme does not use any kind of channel phase information, the beamforming weights that the FAPs apply in transmission become independent of actual channel states. As a consequence, the performance of a multi-antenna system in absence of channel phase information is equivalent to the one of a single-antenna system, where the RVs $X$ and $Y$ are exponentially distributed (with no beamforming gain).

Based on the analysis presented in Appendix 1, we have

$$
F_{Z}(z)=\left[1-e^{-\left(\frac{z}{\bar{\gamma}_{1 \rightarrow 1}}+\frac{\hat{\gamma}_{\text {th }}}{\bar{\gamma}_{2 \rightarrow 1}}\right)}\right]-\frac{\bar{\gamma}_{1 \rightarrow 1}}{\left(\bar{\gamma}_{2 \rightarrow 1} z+\bar{\gamma}_{1 \rightarrow 1}\right)} e^{-\frac{z}{\bar{\gamma}_{1 \rightarrow 1}}}\left[1-e^{-\hat{\gamma}_{\text {th }}\left(\frac{z}{\bar{\gamma}_{1 \rightarrow 1}}+\frac{1}{\bar{\gamma}_{2 \rightarrow 1}}\right)}\right],
$$

where $\hat{\gamma}_{\text {th }}$ is the threshold that defines the SR condition in the cross-link (note that the super-index (c) has been omitted to simplify the notation). As expected, closedform formula (26) reduces to (21) (applying the corresponding substitution of indexes) when the threshold value in the cross-link is set high enough to guarantee a continuous transmission in the interfering femtocell (i.e., when $\hat{\gamma}_{\text {th }} \rightarrow \infty$ ).

It is important to highlight that, the performance of TBF in absence of channel phase information is only identical to the one of a single-antenna system from a theoretical perspective, or when the frequency responses of the channels are strictly flat. Since perfect frequency flat channels do not exist in practice, the incoherent superposition of the copies of the same signal received from the different elements of an antenna array will actually increase the delay spread of the equivalent channel (with respect to the one that would be obtained using only one transmit antenna). Such an approach, that may be useful to reap frequency diversity applying a proper channel coding scheme, lies beyond the scope of this article and is therefore not considered. Nevertheless, it just mentioned here to avoid a wrong interpretation of the concepts presented in this section.

\section{Partial channel phase information}

When the FAP in better condition selects the TBF weights for interference mitigation purposes (i.e., altruistic beamforming), the stochastic behavior of the desired signal and the interference that the FUE in disadvantageous situation perceives can be approximated as $\chi^{2}$ distributed RVs with 4 and 2 degrees of freedom, respectively. 
Based on the analysis presented in Appendix 1, it is possible to see that

$$
\begin{aligned}
F_{Z}(z)= & 1-\left(1+\frac{2 z}{\mathcal{G} \bar{\gamma}_{1 \rightarrow 1}}\right) e^{-\left(\frac{2 z}{\mathcal{G} \bar{\gamma}_{1 \rightarrow 1}}+\frac{\hat{\gamma}_{\text {th }}}{g \bar{\gamma}_{2 \rightarrow 1}}\right)}+\frac{2 z \hat{\gamma}_{\text {th }}}{2 g \bar{\gamma}_{2 \rightarrow 1} z+\mathcal{G} \bar{\gamma}_{1 \rightarrow 1}} e^{-\left[\frac{\left.2 z \hat{\gamma}_{\mathrm{h}}+1\right)}{\mathcal{G} \bar{\gamma}_{1 \rightarrow 1}}+\frac{\hat{\gamma}_{\mathrm{h}}}{g \bar{\gamma}_{2 \rightarrow 1}}\right]} \\
& -\left[\frac{2 z+\mathcal{G} \bar{\gamma}_{1 \rightarrow 1}}{2 g \bar{\gamma}_{2 \rightarrow 1} z+\mathcal{G} \bar{\gamma}_{1 \rightarrow 1}}+\frac{2 z g \mathcal{G} \bar{\gamma}_{1 \rightarrow 1} \bar{\gamma}_{2 \rightarrow 1}}{\left(2 g \bar{\gamma}_{2 \rightarrow 1} z+\mathcal{G} \bar{\gamma}_{1 \rightarrow 1}\right)^{2}}\right] \\
& e^{-\frac{2 z}{\mathcal{G} \bar{\gamma}_{1 \rightarrow 1}}}\left[1-e^{-\hat{\gamma}_{\mathrm{th}}\left(\frac{2 z}{\left.\bar{G} \overline{\gamma_{1 \rightarrow 1}}+\frac{1}{g \bar{\gamma}_{2 \rightarrow 1}}\right)}\right]}\right.
\end{aligned}
$$

Here, the precise values for $\mathcal{G}$ and $g$ are given in (17), and depend on the number of phase bits $N$ that the serving FAP and interfering FAP use, respectively, to identify the beamforming weights that should be applied in transmission.

\section{Full channel phase information (asymptotic upper bound)}

Before finishing this section, we identify the performance of our scheme when the channel phase information is reported to the transmitter without quantization. Note that the full channel phase information case gives the performance upper bound, and allows the provision of a quantitative measure of the impact of channel phase information accuracy in the performance of the method. The main difference between the full and partial channel phase information case is that, in the former one, the stochastic behavior of the interfering signal at the victim mobile user should be approximated as a $\chi^{2}$ distributed RV with 1 degree of freedom. The fading figure value that allows us to arrive to this conclusion can be easily computed using the closed-form expressions derived in Appendix B of [28].

Based on the analysis presented in Appendix 1, it is possible to see that

$$
\begin{aligned}
& F_{Z}(z)=1-\left(1+\frac{2 z}{\mathcal{G} \bar{\gamma}_{1 \rightarrow 1}}\right)\left(\frac{1}{2 g \bar{\gamma}_{2 \rightarrow 1}}\right)^{\frac{1}{2}}\left(\frac{1}{2 g \bar{\gamma}_{2 \rightarrow 1}}+\frac{2 z}{\mathcal{G} \bar{\gamma}_{1 \rightarrow 1}}\right)^{-\frac{1}{2}} \\
& e^{-\frac{2 z}{\mathcal{G} \bar{\gamma}_{1 \rightarrow 1}}} \operatorname{erf}\left(\sqrt{\left(\frac{1}{2 g \bar{\gamma}_{2 \rightarrow 1}}+\frac{2 z}{\mathcal{G} \bar{\gamma}_{1 \rightarrow 1}}\right) \hat{\gamma}_{\text {th }}}\right) \\
& -\frac{2 z}{\mathcal{G} \bar{\gamma}_{1 \rightarrow 1}}\left(\frac{1}{2 \pi g \bar{\gamma}_{2 \rightarrow 1}}\right)^{\frac{1}{2}} e^{-\frac{2 z}{\mathcal{G} \bar{\gamma}_{1 \rightarrow 1}}}\left\{\frac{\sqrt{\pi}}{2}\left(\frac{1}{2 g \bar{\gamma}_{2 \rightarrow 1}}+\frac{2 z}{\mathcal{G} \bar{\gamma}_{1 \rightarrow 1}}\right)^{-\frac{3}{2}}\right. \\
& \operatorname{erf}\left(\sqrt{\left(\frac{1}{2 g \bar{\gamma}_{2 \rightarrow 1}}+\frac{2 z}{\mathcal{G} \bar{\gamma}_{1 \rightarrow 1}}\right) \hat{\gamma}_{\text {th }}}\right)-\sqrt{\hat{\gamma}_{\text {th }}}\left(\frac{1}{2 g \bar{\gamma}_{2 \rightarrow 1}}+\frac{2 z}{\mathcal{G} \bar{\gamma}_{1 \rightarrow 1}}\right)^{-1} \\
& \left.e^{-\left(\frac{1}{2 g \bar{\gamma}_{2 \rightarrow 1}}+\frac{2 z}{\mathcal{G} \bar{\gamma}_{1 \rightarrow 1}}\right) \hat{\gamma}_{\text {th }}}\right\}-\left(1+\frac{2 z}{\mathcal{G} \bar{\gamma}_{1 \rightarrow 1}}\right) e^{-\frac{2 z}{\mathcal{G} \bar{\gamma}_{1 \rightarrow 1}}} \operatorname{erfc}\left(\sqrt{\frac{\hat{\gamma}_{\text {th }}}{2 g \bar{\gamma}_{2 \rightarrow 1}}}\right)
\end{aligned}
$$

is valid in this situation, providing the upper bound performance of the system when the number of phase bits becomes large (i.e., when $N \rightarrow \infty$ ).

\section{Scheduling of silence periods based on direct-link signal strength}

In this section, we derive a closed-form expression to model the received SINR at the victim FUE, when the inter-cell scheduling between femtocells is based on the strength of its direct-link (i.e., SRs are triggered if the strength of the victim's direct-link is not good enough). When combining TBF with channel-aware scheduling of SPs according to (10), the CDF expression presented in (12) becomes

$$
F_{Z}(z)=F_{Z}\left(z \mid X>\check{\gamma}_{\text {th }}\right) \operatorname{Pr}\left\{X>\check{\gamma}_{\text {th }}\right\}+F_{X}\left(z \mid X \leq \check{\gamma}_{\text {th }}\right) \operatorname{Pr}\left\{X \leq \check{\gamma}_{\text {th }}\right\} \quad z \geq 0 .
$$


Note that the second term of (29) characterize the SINR at the victim FUE when the interfering FAP is providing a SP. So, the CDF that is presented in the second term of (29) is actually the one that models the received SNR from the serving FAP (i.e., the CDF of $\mathrm{RV} X$, because there is no interference originated in the neighboring FAP in this case).

\section{Single-antenna system (no channel phase information)}

In absence of channel phase information, the stochastic behavior of the desired signal energy (i.e., RV $X$ ) and the co-channel interference (i.e., RV $Y$ ) can be modeled as an exponentially distributed RV (see Section 'Scheduling of silence periods based on cross-link signal strength' for more details). Then, based on the analysis presented in Appendix 2, we have

$$
\begin{aligned}
F_{Z}(z)= & e^{-\frac{\check{y}_{\text {th }}}{\bar{\gamma}_{1 \rightarrow 1}}} e^{-\frac{\max \left\{\frac{\check{y}_{\text {th }}}{z}-1,0\right\}}{\bar{\gamma}_{2 \rightarrow 1}}}+1-e^{-\frac{\min \left\{z, \check{\gamma}_{\text {th }}\right\}}{\bar{\gamma}_{1 \rightarrow 1}}} \\
& -e^{-\frac{z}{\bar{\gamma}_{1 \rightarrow 1}}}\left(\frac{\bar{\gamma}_{1 \rightarrow 1}}{z \bar{\gamma}_{2 \rightarrow 1}+\bar{\gamma}_{1 \rightarrow 1}}\right) e^{-\left(\frac{z}{\bar{\gamma}_{1 \rightarrow 1}}+\frac{1}{\bar{\gamma}_{2 \rightarrow 1}}\right) \max \left\{\frac{\check{\gamma}_{\text {th }}}{z}-1,0\right\}} .
\end{aligned}
$$

Note that in the previous equation, $\check{\gamma}_{\text {th }}$ represents the threshold that defines the SR condition in the direct-link. Again, super-index $(d)$ is omitted to simplify the notation.

\section{Partial channel phase information}

In presence of partial channel phase information, the stochastic behavior of the desired signal energy (i.e., $\mathrm{RV} X$ ) and the co-channel interference (i.e., RV $Y$ ) can be approximated as a $\chi^{2}$ distributed RV with 4 and 2 degrees of freedom, respectively (see Section 'Schedul ing of silence periods based on cross-link signal strength' for more details). Then, based on the analysis presented in Appendix 2, it is found that

$$
\begin{aligned}
& F_{Z}(z)=\left(1+\frac{2 \check{\gamma}_{\text {th }}}{\mathcal{G} \bar{\gamma}_{1 \rightarrow 1}}\right) e^{-\frac{2 \check{\gamma}_{\text {th }}}{\mathcal{G} \bar{\gamma}_{1 \rightarrow 1}}} e^{-\frac{\max \left\{\frac{\check{\gamma}_{\text {th }}}{z}-1,0\right\}}{g \bar{\gamma}_{2} \rightarrow 1}}+1-\left(1+\frac{2 \min \left\{z, \check{\gamma}_{\text {th }}\right\}}{\mathcal{G} \bar{\gamma}_{1 \rightarrow 1}}\right) e^{-\frac{2 \min \left\{z \check{\gamma}_{\text {th }}\right\}}{\mathcal{G} \bar{\gamma}_{1}}} \\
& -\left(1+\frac{2 z}{\mathcal{G} \bar{\gamma}_{1 \rightarrow 1}}\right)\left(\frac{1}{g \bar{\gamma}_{2 \rightarrow 1}}\right)\left(\frac{2 z}{\mathcal{G} \bar{\gamma}_{1 \rightarrow 1}}+\frac{1}{g \bar{\gamma}_{2 \rightarrow 1}}\right)^{-1}
\end{aligned}
$$

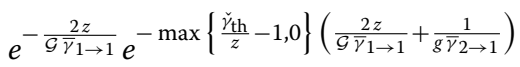

$$
\begin{aligned}
& -\left(\frac{2 z}{\mathcal{G} \bar{\gamma}_{1 \rightarrow 1}}\right)\left(\frac{1}{g \bar{\gamma}_{2 \rightarrow 1}}\right)\left(\frac{2 z}{\mathcal{G} \bar{\gamma}_{1 \rightarrow 1}}+\frac{1}{g \bar{\gamma}_{2 \rightarrow 1}}\right)^{-2} e^{-\frac{2 z}{\mathcal{G} \bar{\gamma}_{1 \rightarrow 1}}} \\
& \times e^{-\max \left\{\frac{\check{\gamma}_{\text {th }}}{z}-1,0\right\}\left(\frac{2 z}{\mathcal{G} \bar{\gamma}_{1 \rightarrow 1}}+\frac{1}{g \bar{\gamma}_{2 \rightarrow 1}}\right)}\left[\max \left\{\frac{\check{\gamma}_{\text {th }}}{z}-1,0\right\}\left(\frac{2 z}{\mathcal{G} \bar{\gamma}_{1 \rightarrow 1}}+\frac{1}{g \bar{\gamma}_{2 \rightarrow 1}}\right)+1\right],
\end{aligned}
$$

where the precise values for the beamforming gains $\mathcal{G}$ and $g$ depend on the number of phase bits $N$ that are used to identify the beamforming weights at both FAPs (i.e., the serving FAP and the interfering FAP).

\section{Full channel phase information (asymptotic upper bound)}

Finally, we aim to model the performance of the transmission scheme when the channel phase information is reported to the transmitter with no quantization. In this case, the stochastic behavior of the desired signal energy (i.e., RV X) and the co-channel interference (i.e., RV $Y$ ) can be approximated as a $\chi^{2}$ distributed RV with 4 and 1 degrees of freedom, respectively (see Section 'Scheduling of silence periods based on cross-link signal strength' for more details). 
Then, based on the analysis presented in Appendix 2, it is possible to see that

$$
\begin{aligned}
& F_{Z}(z)=\left(1+\frac{2 \check{\gamma}_{\text {th }}}{\mathcal{G} \bar{\gamma}_{1 \rightarrow 1}}\right) e^{-\frac{2 \check{\gamma}_{\text {th }}}{\mathcal{G} \bar{\gamma} \bar{\gamma}_{1 \rightarrow 1}} \operatorname{erfc}}\left(\sqrt{\frac{\max \left\{\frac{\check{\gamma}_{\text {th }}}{z}-1,0\right\}}{2 g \bar{\gamma}_{2 \rightarrow 1}}}\right) \\
& -\left(1+\frac{2 z}{\mathcal{G} \bar{\gamma}_{1 \rightarrow 1}}\right) \sqrt{\frac{\mathcal{G} \bar{\gamma}_{1 \rightarrow 1}}{4 z g \bar{\gamma}_{2 \rightarrow 1}+\mathcal{G} \bar{\gamma}_{1 \rightarrow 1}}} e^{-\frac{2 z}{\mathcal{G} \bar{\gamma}_{1 \rightarrow 1}}} \\
& \operatorname{erfc}\left(\sqrt{\max \left\{\frac{\check{\gamma}_{\text {th }}}{z}-1,0\right\}\left(\frac{2 z}{\mathcal{G} \bar{\gamma}_{1 \rightarrow 1}}+\frac{1}{2 g \bar{\gamma}_{2 \rightarrow 1}}\right)}\right) \\
& -\left(\frac{2 z}{\mathcal{G} \bar{\gamma}_{1 \rightarrow 1}}\right) \frac{1}{\sqrt{2 \pi g \bar{\gamma}_{2 \rightarrow 1}}} e^{-\frac{2 z}{\mathcal{G} \bar{\gamma}_{1 \rightarrow 1}}} \\
& \times\left\{\frac{\sqrt{\max \left\{\frac{\check{\gamma}_{\text {th }}}{z}-1,0\right\}}}{\left(\frac{2 z}{\mathcal{G} \bar{\gamma}_{1 \rightarrow 1}}+\frac{1}{2 g \bar{\gamma}_{2 \rightarrow 1}}\right)} e^{-\max \left\{\frac{\check{\gamma}_{\text {th }}}{z}-1,0\right\}\left(\frac{2 z}{\mathcal{G} \bar{\gamma}_{1 \rightarrow 1}}+\frac{1}{2 g \bar{\gamma}_{2 \rightarrow 1}}\right)}\right. \\
& \left.+\frac{\sqrt{\pi}}{2\left(\frac{2 z}{\mathcal{G} \bar{\gamma}_{1 \rightarrow 1}}+\frac{1}{2 g \bar{\gamma}_{2 \rightarrow 1}}\right)^{\frac{3}{2}}} \operatorname{erfc}\left[\sqrt{\max \left\{\frac{\check{\gamma}_{\text {th }}}{z}-1,0\right\}\left(\frac{2 z}{\mathcal{G} \bar{\gamma}_{1 \rightarrow 1}}+\frac{1}{2 g \bar{\gamma}_{2 \rightarrow 1}}\right)}\right]\right\} \\
& +1-\left(1+\frac{2 \min \left\{z, \check{\gamma}_{\mathrm{th}}\right\}}{\mathcal{G} \bar{\gamma}_{1 \rightarrow 1}}\right) e^{-\frac{2 \min \left\{z \check{\gamma}_{\mathrm{h}}\right\}}{\mathcal{G} \bar{\gamma}_{1 \rightarrow 1}}}
\end{aligned}
$$

is valid in this situation, providing the upper bound performance of the system when the number of phase bits becomes large (i.e., when $N \rightarrow \infty$ ).

\section{Performance analysis}

In this section, we analyze the performance of TBF when combined with SR-based channel-aware scheduling. To illustrate the performance in each case, we utilize the CDFs of the achievable spectral efficiency and the outage rates that are observed in the different situations.

\section{Cumulative distribution function}

Since our aim is to equalize the rates of the two femtocells, let us first focus our attention in an unbalanced user scenario. Therefore, we analyze the rate performance when the FUE in the first femtocell experiences a good signal strength (e.g., $\bar{\gamma}_{1 \rightarrow 1}=15 \mathrm{~dB}$ ), but at the same time the interfering signal originated in the second femtocell is also strong (e.g., $\bar{\gamma}_{2 \rightarrow 1}=15 \mathrm{~dB}$ ). Simultaneously, the FUE being served by the second femtocell experiences a very good signal strength (e.g., $\bar{\gamma}_{2 \rightarrow 2}=20 \mathrm{~dB}$ ), but the strength of the interfering signal originated in the first femtocell is weak (e.g., $\bar{\gamma}_{1 \rightarrow 2}=0 \mathrm{~dB}$ ). Note that the background noise power $P_{N}$ is used as reference to measure the signal strengths in the different links. As expected, in this case one user receives on average a significantly higher SINR as indicated by the solid lines in Figure 2 (i.e., when no TBF method is applied in transmission).

When both users start to apply 2-antenna egoistic beamforming (dashed lines), both users gain, but the performance gap remains. However, as FAP 2 selects its beamforming vector to minimize interference to FUE 1, FUE 2 loses its coherent combining gain (moving back to the solid line), but FUE 1 gains from receiving less interference (dashed- 


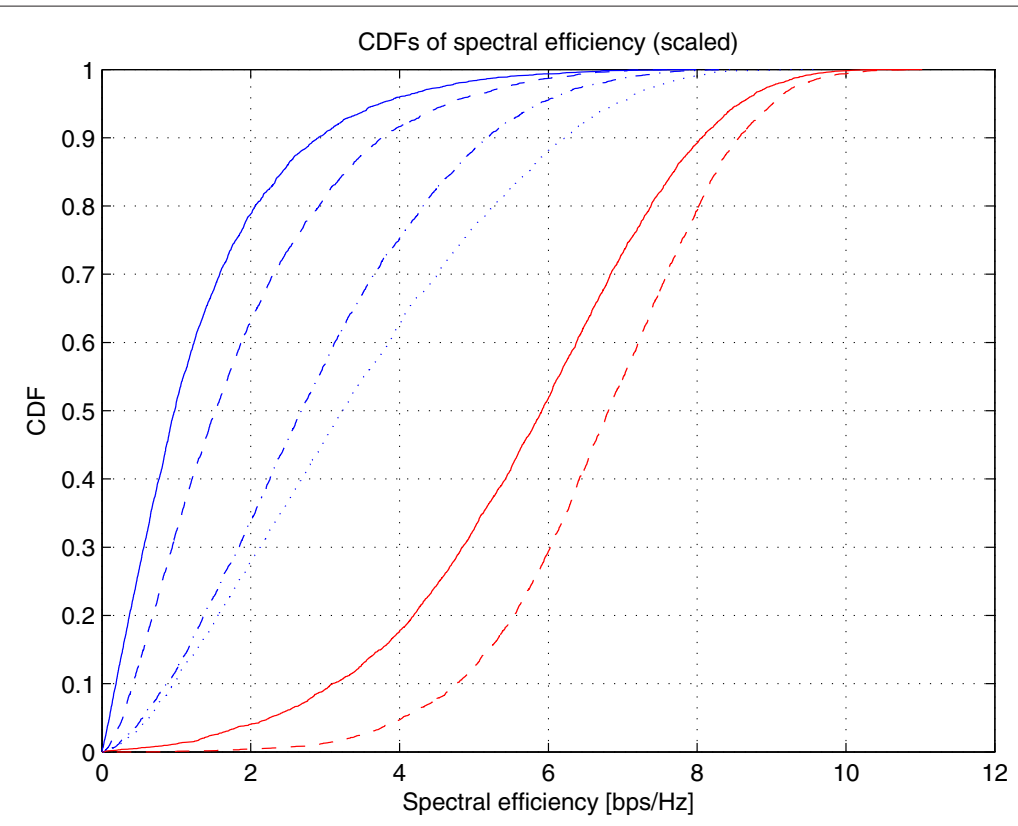

Figure 2 Rate cumulative distribution function for FUE 1 (blue) and FUE 2 (red) when 2-bit mode 1 is applied with different beamforming methods (no SP scheduling implemented). Interference scenario: $\bar{\gamma}_{1 \rightarrow 1}=15 \mathrm{~dB}$ (good signal), $\bar{\gamma}_{1 \rightarrow 2}=0 \mathrm{~dB}$ (weak interference), $\bar{\gamma}_{2 \rightarrow 1}=15 \mathrm{~dB}$ (strong interference), and $\bar{\gamma}_{2 \rightarrow 2}=20 \mathrm{~dB}$ (excellent signal). All cases: $P_{N}$ used as reference power. Solid: No TBF. Dashed: 2-bit mode 1 (FAP 1 ego/FAP 2 ego). Dashed-dotted: 2 -bit mode 1 (FAP 1 ego/FAP 2 alt). Dotted: $\infty$-bit mode 1 (FAP 1 ego/FAP 2 alt).

dotted line). Finally, FUE 1 can further reduce the gap when more bits are allocated for TBF purposes at both FAPs (FUE 1: dotted line FUE 2: solid line). It is important to highlight that even when $N \rightarrow \infty$, the interference generated at FAP 2 will not completely be nulled at victim FUE 1 (even when both signals are put exactly in phase opposition, some residual interference will remain since the transmit power cannot be adjusted individually at each antenna in our system model). We note that the closed-form expression for the performance of $\infty$-bit mode 1 (dotted line) can be obtained from (28), setting the threshold value in the cross-link high enough to guarantee a continuous transmission in FAP 2. As expected, the same closed-form expression is obtained from (32) when the threshold value in the direct-link tends to zero.

As seen in Figure 2, TBF can effectively narrow down the performance gap of the two users, but some difference still remains. Let us next look at the performance of the proposed scheduling method for closing the remaining gap. The solid lines in Figure 3 correspond to the situation in which 2-bit mode 1 altruistic beamforming has already been applied in FAP 2 for interference mitigation purposes at FUE 1. Now, using SRbased channel-aware scheduling we can provide to FUE 1 periods of low interference, increasing as a consequence the likelihood of experiencing higher instantaneous data rates. However, the selection of the applied threshold affects significantly the SINR region in which the (opportunistic) scheduling gain will appear.

According to the results presented in Figure 3, it is clear that all kind of thresholds practically balance the rates of the two users when the interfering FAP 2 is able to reduce its transmission cycle, to improve the situation of victim FUE 1. Concretely, in this case we assume that FAP 2 receives SRs (and guarantee SPs) in 30\% of the time frames. We 


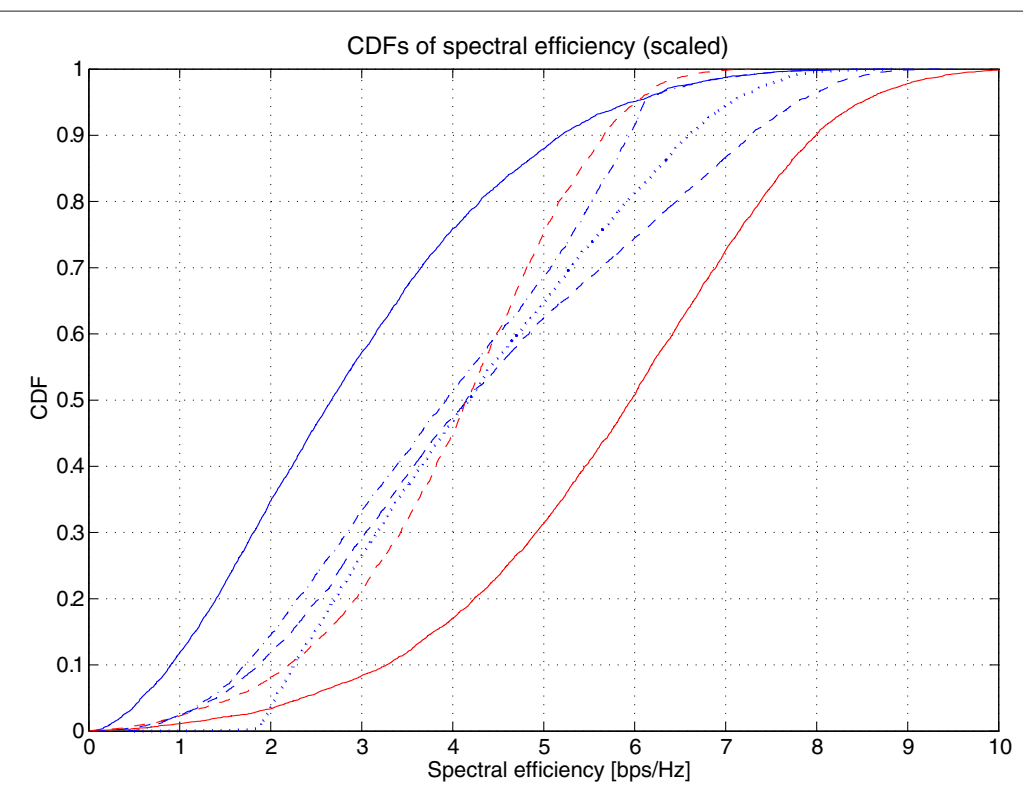

Figure 3 Rate cumulative distribution function for FUE 1 (blue) and FUE 2 (red) when 2-bit mode 1 is applied (FAP 1 ego/FAP 2 alt). Interference scenario: $\bar{\gamma}_{1 \rightarrow 1}=15 \mathrm{~dB}, \bar{\gamma}_{1 \rightarrow 2}=0 \mathrm{~dB}, \bar{\gamma}_{2 \rightarrow 1}=15 \mathrm{~dB}$, and $\bar{\gamma}_{2 \rightarrow 2}=20 \mathrm{~dB}$. All cases: $P_{N}$ used as reference power. Solid curves: constant transmission (i.e., no scheduling). Blue curves: dashed $\left(\hat{\gamma}_{\text {th }}^{(c)}\right)$, dashed-dotted $\left(\check{\gamma}_{\text {th }}^{(d)}\right)$, dotted $\left(\check{\gamma}_{\text {th }}^{(\Upsilon)}\right)$. Dashed red: all kinds of thresholds. Threshold selection: FUE 2 is $30 \%$ of time silent.

note that here, the selection of this specific activity factor is just for illustrative purposes (i.e., similar results are expected to be observed for different percentages of SRs). Studying the CDF regions in more detail it can be noticed that, setting the threshold on the instantaneous received SINR $\check{\gamma}_{\text {th }}^{(\Upsilon)}$ provides the best performance at working regimes with low spectral efficiencies (i.e., at low outage probabilities). When implementing a channel-aware scheduling algorithm based on $\check{\gamma}_{\text {th }}^{(\Upsilon)}$, FUE 1 issues a SR in those time instants where its instantaneous received SINR is below a predefined threshold (like in previous cases, the threshold should be selected to guarantee the percentage of SPs that FAP 2 agreed in advance to support). On the other hand, the scheduling of SPs using the cross-link threshold $\hat{\gamma}_{\text {th }}^{(c)}$ provides a better performance at working regimes with high spectral efficiencies (i.e., at high outage probabilities). Finally, the implementation of a channel-aware scheduling algorithm that relies on the direct-link threshold $\check{\gamma}_{\text {th }}^{(d)}$ provides the worst spectral efficiency over the whole CDF region. Note that the scheduling of SPs based on $\check{\gamma}_{\text {th }}^{(\Upsilon)}$ is included in this section for illustrative purposes, and its performance is solely evaluated based on numerical simulations. In addition, it is important to highlight that the spectral efficiencies in the second femtocell (i.e., red curves in Figure 3) are scaled by the corresponding activity factor that interfering FAP 2 agreed to support in advance with FAP 1.

Last but not least, let us look at the effect of the background noise-like interference on the performance of the algorithm that schedules SPs using the cross-link threshold $\hat{\gamma}_{\text {th }}^{(c)}$. To carry out this analysis, we first select a value of background noise power $P_{N}$ as reference, and then we define the mean received SNR values for the different links in $\mathrm{dBs}$, using (14). After that, we start to adjust the power of the background noise in $\Delta \mathrm{dB}$, keeping the rest of the parameters of the system constant (i.e., transmit powers and path 
loss attenuations). Three cases are going to be analyzed: $\Delta=10 \mathrm{~dB}$ (strong background noise regime), $\Delta=0 \mathrm{~dB}$ (reference background noise regime), and $\Delta=-10 \mathrm{~dB}$ (weak background noise regime). Figure $4 \mathrm{a}$ shows that the combination of TBF and channelaware scheduling of SPs balances the rates effectively, except for the low background noise case (dotted line). This result is based on the fact that, as the system becomes more isolated (i.e., the background noise decreases), FUE 1 is able to receive very high SINR values during the SPs of FAP 2. The same behavior can be observed in Figure 4b, in which both FAPs apply an egoistic beamforming scheme. Nevertheless, it is evident that in the absence of interference mitigation gain (i.e., when both FAPs behave egoistically), the rates in the system do not remain balanced. In fact, equalizing the rates at the 50th percentile would require the interfering femtocell to be silent in approximately $40-45 \%$ of the transmission time instants (depending on the background noise level).

\section{Outage rate}

In addition to the CDF approach presented in the previous section, we investigate the system performance in terms of outage rates. The outage rate of a system can be defined as:

$$
R^{\text {out }}\left(P^{\text {out }}\right)=\log _{2}\left(1+\gamma\left(P^{\text {out }}\right)\right),
$$

where $\gamma\left(P^{\text {out }}\right)$ is the SINR needed to achieve a given outage probability $P^{\text {out }}$. The value of $\gamma\left(P^{\text {out }}\right)$, can be obtained by computing the solution for the following equation:

$$
P^{\text {out }}=\operatorname{Pr}\left\{\log _{2}(1+\gamma)<R^{\text {out }}\right\}=\int_{0}^{\gamma_{R^{\text {out }}}} f_{\Upsilon}(\gamma) d \gamma=F_{\Upsilon}\left(\gamma_{R^{\text {out }}}\right) .
$$

Let us now look at the corresponding rates at outage probabilities 10 and $50 \%$, when the threshold value $\widehat{\gamma}_{\text {th }}$ (or $\left.\check{\gamma}_{\text {th }}\right)$ varies. As seen in Figure 5, TBF mode 1 provides a significant gain, but the relative gain decreases as the number of phase bits increases. Comparing

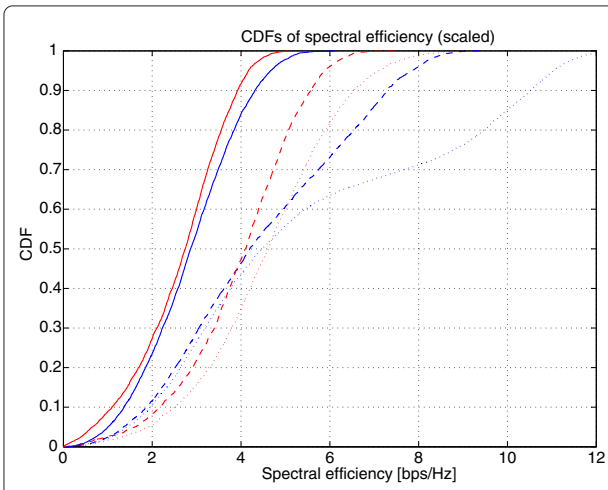

(a) (FAP 1 ego/FAP 2 alt)

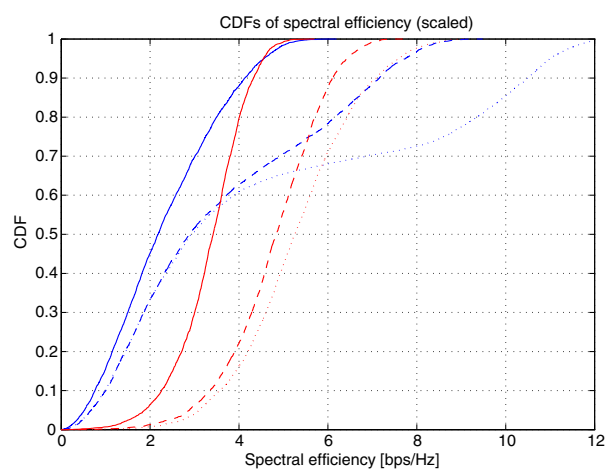

(b) (FAP 1 ego/FAP 2 ego)

Figure 4 Rate cumulative distribution function for FUE 1 (blue) and FUE 2 (red) when 2-bit mode 1 is applied with different beamforming methods and various background noise regimes. (a) (FAP 1 ego/FAP 2 alt). (b) (FAP 1 ego/FAP 2 ego). Interference scenario: $\bar{\gamma}_{1 \rightarrow 1}=(15-\Delta) \mathrm{dB}, \bar{\gamma}_{1 \rightarrow 2}=(0-\Delta) \mathrm{dB}$, $\bar{\gamma}_{2 \rightarrow 1}=(15-\Delta) \mathrm{dB}$, and $\bar{\gamma}_{2 \rightarrow 2}=(20-\Delta) \mathrm{dB}$. All cases: $P_{N}$ used as reference power. Solid: $\Delta=10 \mathrm{~dB}$ (strong background noise regime). Dashed: $\Delta=0 \mathrm{~dB}$ (reference background noise regime). Dotted: $\Delta=-10 \mathrm{~dB}$ (weak background noise regime). Scheduling criterion: $\hat{\gamma}_{\text {th }}^{(c)}$ (all cases). Threshold selection: FUE 2 is $30 \%$ of time silent. 


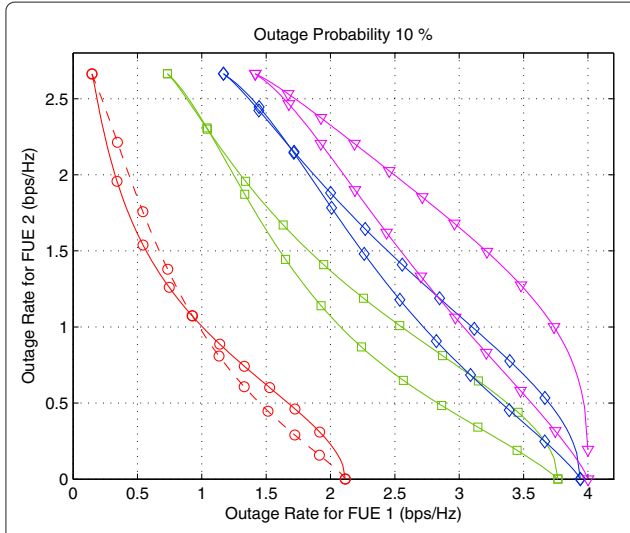

(a) 10-th percentile outage rate

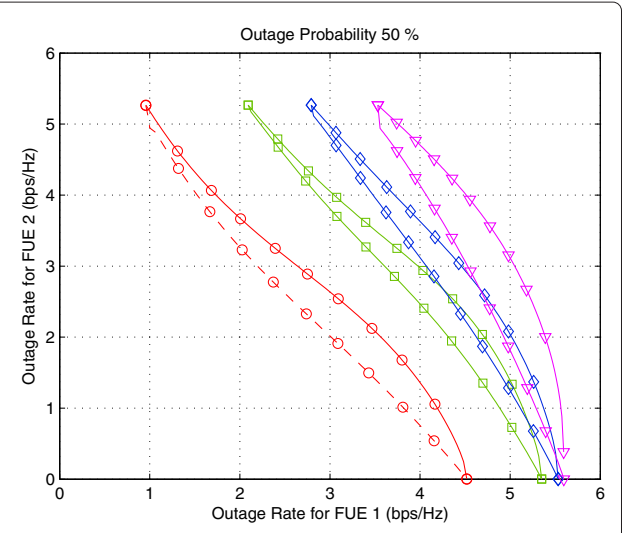

(b) 50-th percentile outage rate

Figure 5 Rate region for outage probability $\mathbf{1 0} \%$ and $\mathbf{5 0 \%}$. Red (o): Single-antenna. Green ( $\square$ ): 1-bit mode 1. Blue $(\diamond): 2$-bit mode 1. Magenta $(\nabla): \infty$-bit mode 1. Solid line: $\hat{\gamma}_{\text {th }}^{(c)}$. Dashed line: $\check{\gamma}_{\text {th }}^{(d)}$. Interference scenario: $\bar{\gamma}_{1 \rightarrow 1}=15 \mathrm{~dB}, \bar{\gamma}_{1 \rightarrow 2}=0 \mathrm{~dB}, \bar{\gamma}_{2 \rightarrow 1}=15 \mathrm{~dB}$, and $\bar{\gamma}_{2 \rightarrow 2}=20 \mathrm{~dB}$. All cases: $P_{N}$ used as reference power.

the performance when using the threshold on the interference link $\hat{\gamma}_{\text {th }}^{(c)}$ and on the directlink $\check{\gamma}_{\text {th }}^{(d)}$, it can be concluded that the usage of cross-link threshold provides superior performance, except for the single-antenna case with $10 \%$ outage probability. In addition, it is worth noting that in all cases, the rates achieved in both femtocells can be balanced by selecting an appropriate threshold value for the scheduling of SPs at the interfering FAP.

Related to the aim of balancing the data rates of the femtocells, let us next analyze the max-min outage rates when combining TBF and channel-aware scheduling (solid lines in Figure 6), i.e.,

$$
R_{\max -\min }^{\text {out }}=\max _{\hat{\gamma}_{\text {th }}}\left\{\min \left\{R_{1}^{\text {out }}\left(\hat{\gamma}_{\mathrm{th}}\right), R_{2}^{\text {out }}\left(\hat{\gamma}_{\mathrm{th}}\right)\right\}\right\},
$$

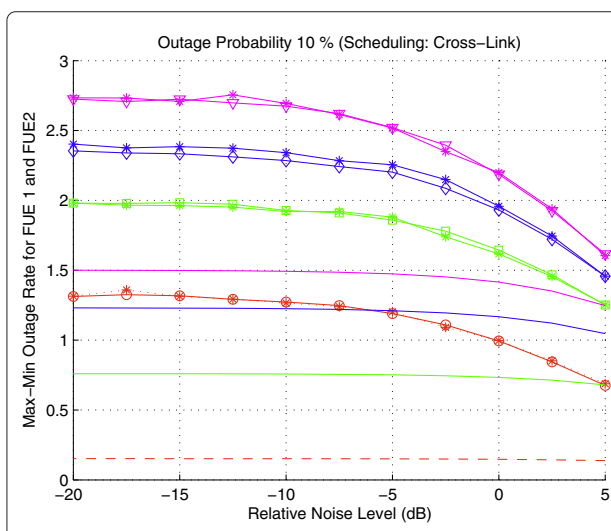

(a) 10-th percentile outage rate

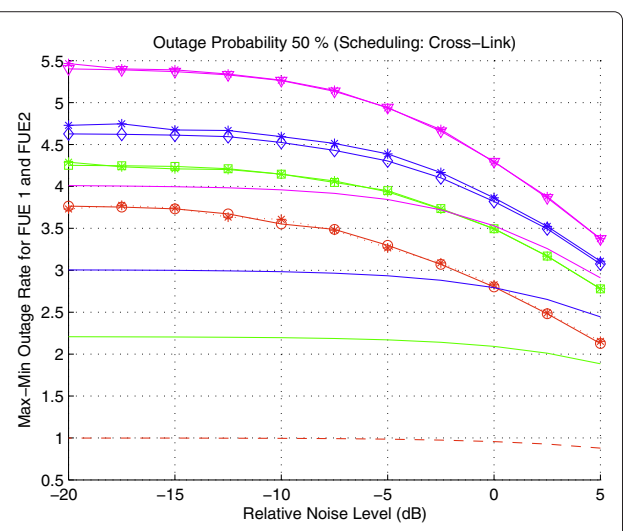

(b) 50-th percentile outage rate

Figure 6 Max-min and min rates for outage probability $\mathbf{1 0} \%$ and $\mathbf{5 0 \%}$. Red (o): Single-antenna. Green ( $\square)$ : 1-bit mode 1. Blue ( $\diamond): 2$-bit mode 1. Magenta $(\nabla): \infty$-bit mode 1. Solid line: TBF plus channel-aware scheduling using $\widehat{\gamma}_{\text {th }}^{(c)}$ (all cases). Dashed line: only TBF (min outage rate). Interference scenario: $\bar{\gamma}_{1 \rightarrow 1}=(15-\Delta) \mathrm{dB}, \bar{\gamma}_{1 \rightarrow 2}=(0-\Delta) \mathrm{dB}, \bar{\gamma}_{2 \rightarrow 1}=(15-\Delta) \mathrm{dB}$, and $\bar{\gamma}_{2 \rightarrow 2}=(20-\Delta) \mathrm{dB}$. All cases: $P_{N}$ used as reference power. Point values (" $*$ ") were simulated to verify the analytical results. 
and the equivalent min outage rates in presence of TBF only (dashed lines in Figure 6), i.e.,

$$
R_{\text {min }}^{\text {out }}=\min \left\{R_{1}^{\text {out }}\left(\hat{\gamma}_{\text {th }} \rightarrow \infty\right), R_{2}^{\text {out }}\left(\hat{\gamma}_{\text {th }} \rightarrow \infty\right)\right\}
$$

Looking at the $10 \%$ outage probability case (i.e., Figure $6 \mathrm{a}$ ) and $50 \%$ outage probability case (i.e., Figure $6 \mathrm{~b}$ ), we observe that the combination of TBF and channel-aware scheduling provides clearly the highest performance. Nevertheless, as the relative noise level increases, the gain from scheduling decreases as well. This decrease is significantly steeper than in the case where only TBF is applied. At the $50 \%$ outage rate level, the trends are very similar to the $10 \%$ case, but the rates are obviously higher. Still, it is important to note that already 1-bit mode 1 provides a remarkable gain with and without scheduling (using the case with no channel information as baseline) and could, therefore, significantly reduce outage in a practical system. In general, the more isolated the system is (i.e., the smaller is the background noise power) the better the proposed combination of TBF and channel-aware scheduling of SPs works.

Finally, the interference link strength greatly affects the performance of the proposed TBF and scheduling methods, as illustrated in Figure 7. Eventhough the rates logically decrease as the interference link becomes stronger, the achievable gain from scheduling also increases while the beamforming gain is close to the same magnitude. However, it is worth noting that when the interference link is weak, allocating more than 1 phase bit for TBF does not result in a significant performance gain. Generally, as noticed in Figure 7, the proposed TBF and scheduling methods provide a substantial gain in terms of max-min outage rate although their relative effectiveness varies with the noise level.

Based on the performance analysis of this section, we can conclude that the proposed methods provide significant gains in all analyzed situations. In practical systems, however, there exists a tradeoff between the amount of signaling information and the achievable data rate in downlink. In general, the proposed 1-bit scheduling method provides very remarkable gains when the criterion is max-min rate. However, if we had focused

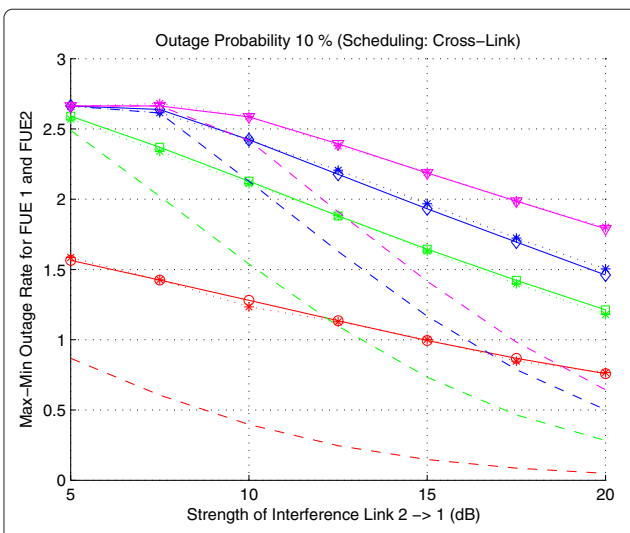

(a) 10-th percentile outage rate

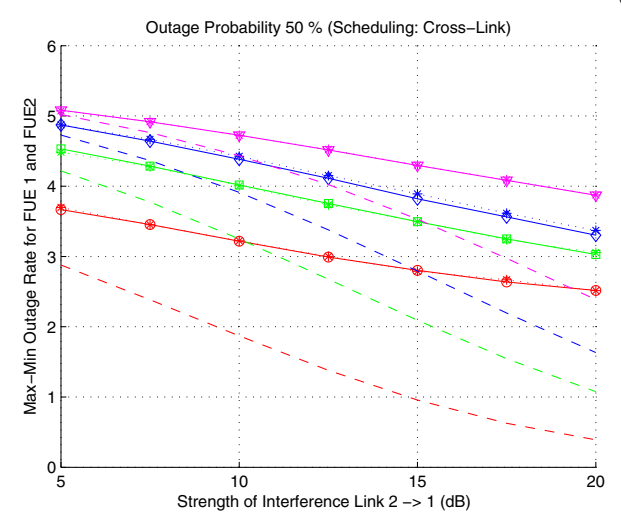

(b) 50-th percentile outage rate

Figure 7 Max-min and min rates for outage probability $\mathbf{1 0} \%$ and $\mathbf{5 0} \%$. Red (o): Single-antenna. Green ( $\square$ ): 1-bit mode 1. Blue $(\diamond)$ : 2-bit mode 1. Magenta $(\nabla): \infty$-bit mode 1. Solid line: TBF plus channel-aware scheduling using $\widehat{\gamma}_{\text {th }}^{(c)}$ (all cases). Dashed line: only TBF (min outage rate). Interference scenario: $\bar{\gamma}_{1 \rightarrow 1}=15 \mathrm{~dB}, \bar{\gamma}_{1 \rightarrow 2}=0 \mathrm{~dB}$, and $\bar{\gamma}_{2 \rightarrow 2}=20 \mathrm{~dB}$. All cases: $P_{N}$ used as reference power. Point values (" $*$ ") were simulated to verify the analytical results. 
on maximizing the sum rate, the conclusions would have been different. In regard to beamforming, it appears that allocating more than two phase bits would not improve the performance enough, to justify the increased amount of signaling information that would be required.

\section{Conclusions and discussion}

Among other interference scenarios, downlink femto-femto co-channel interference has been identified as a key problem related to the deployment of femtocells. Eventhough the received signal power from the serving femto access point (FAP) is typically high, the proximity of neighboring femtocells may lead to high levels of received interference and, as a consequence, low SINR values. In this article, we proposed a combined low-rate intercell transmit beamforming (TBF) and channel-aware scheduling of silence periods (SPs) for both, interference mitigating and data-rate balancing between two femtocells via resource sharing. The implementation of this method requires a low-rate control channel between the victim (interfered) femto user equipment (FUE) and the interfering FAP.

The results indicate that the proposed method is effective to tackle the co-channel interference scenario presented in this article. It is important to highlight that this method allows significant flexibility in system design. First, scheduling requests can only be sent and accepted whenever is necessary, depending on the power (im)balance situation between the two neighboring femtocells. Second, the TBF scheme can be performed in an egoistic or altruistic manner independently at each FAP, according to the SINR situation of the FUE that is served in each femtocell. Finally, the number of bits used for TBF gives flexibility related to design of the control channel for signaling purposes.

In addition to the size of the beamforming codebook, several different scheduling criteria were investigated. Drawing from the results that were presented in Section 'Performance analysis', which were based mainly on closed-form expressions that were derived in Section 'Theoretical analysis', it can be concluded that using the strength of the cross-link as the scheduling criterion provides the best overall performance. However, if the goal is to minimize the occurrence of very low SINR values, setting the threshold on the instantaneous received SINR may lead to better performance results.

The performance of the algorithm was investigated as a function of several variables. It was shown that the proposed methods provide good performance in both, the isolated case (i.e., with only two femtocells present) and in the non-isolated case (i.e., with higher levels of background interference plus noise originated at several neighboring femtocells and/or macrocells to whom interference coordination cannot be implemented). Additionally, the method provides a significant performance gain with varying interference link strengths. As expected, the more isolated the system is, the higher is the relative gain that the proposed method provides. A similar behavior can be observed with respect to the strength of the cross-link, whose value is proportional to the level of interference that is generated between neighboring femtocells.

From the practical implementation point of view there are a few key issues that need to be addressed. First, the method relies on a control-only connection over the air in the cross-link, which should be included in 3GPP standardization to implement the proposed interference coordination method when dealing with closed-access FAPs (the exchange of control information over an X2 like interface seems more reasonable when FAPs belong to the same closed subscriber group). Second, in order to balance the rates, the two FAPs 
should be aware of the current rates that are supported at both femtocells, to be able to calculate the corresponding scheduling threshold value and choose the number of bits for TBF purposes (along with the corresponding egoistic/altruistic beamforming strategy). In a practical system, however, this could be handled so that the victim user sends a silence request (SR) and the selected TBF weight information (for interference mitigation) whenever the strength of the cross-link is high, and the interfering FAP decides on the interference mitigation strategy to be applied independently (i.e., based on the requirements of the FUE that it serves). The optimality of the proposed method greatly depends on the selected criterion (e.g., max-min rate or sum rate) and is, to a large extent, a practical system design issue.

The topic investigated here calls for more research. In this article, we evaluated several different and independent intercell scheduling criteria. In some cases, different criteria could be used simultaneously to improve the average performance of the method. In addition, an algorithm could be developed for making joint decisions related to TBF and channel-aware scheduling strategies. Finally, the methods presented here focused on a pair of femtocells. Further research could address the best way to select these groups of cooperating femtocells, to guarantee coexistence with acceptable complexity requirements when co-located FAPs operate jointly over the same geographic area.

\section{Appendices}

\section{Derivation of Closed-Form CDF expressions}

Here we derive the closed-form CDF expressions, presented in Section 'Theoretical analysis' to study the performance of different interference coordination approaches for femtocells that combine

(a) Various channel phase resolutions to apply TBF, and

(b) Different SP scheduling approaches for interference mitigation.

\section{Appendix 1}

Scheduling of silence periods based on cross-link signal strength

When combining TBF with channel-aware scheduling based on the strength of the crosslink, see (8), the CDF of the received SINR attains the form

$$
F_{Z}(z)=\int_{0}^{\hat{\gamma}_{\text {th }}} F_{X}(z(t+1)) f_{Y}(t) d t+F_{X}(z) \operatorname{Pr}\left\{y>\hat{\gamma}_{\text {th }}\right\} \quad z \geq 0
$$

where different probabilistic models are used to approximate the stochastic behavior of RVs $X$ and $Y$, according to the number of phase bits $N$ that are used in the quantization set of the TBF scheme. In this situation, $\hat{\gamma}_{\text {th }}$ represents the threshold that defines the SR condition in the cross-link.

\section{Single-antenna system (no channel phase information)}

In absence of channel phase information, the stochastic behavior of RVs $X$ and $Y$ can be approximated as exponentially distributed, i.e.,

$$
F_{X}(x)=1-e^{-\frac{x}{\bar{\gamma}_{1 \rightarrow 1}}} \quad x \geq 0, \quad f_{Y}(y)=\frac{1}{\bar{\gamma}_{2 \rightarrow 1}} e^{-\frac{y}{\bar{\gamma}_{2} \rightarrow 1}} \quad y \geq 0 .
$$


Then, combining (37) with (38), we find that

$$
\begin{aligned}
& F_{Z}(z)=\int_{0}^{\hat{\gamma}_{\text {th }}}\left[1-e^{-\frac{z(t+1)}{\bar{\gamma}_{1 \rightarrow 1}}}\right] \frac{1}{\bar{\gamma}_{2 \rightarrow 1}} e^{-\frac{t}{\bar{\gamma}_{2 \rightarrow 1}}} d t+\left[1-e^{-\frac{z}{\bar{\gamma}_{1 \rightarrow 1}}}\right] e^{-\frac{\hat{\gamma}_{\text {th }}}{\bar{\gamma}_{2 \rightarrow 1}}} \\
& =\int_{0}^{\hat{\gamma}_{\mathrm{th}}} \frac{1}{\overline{\bar{\gamma}}_{2 \rightarrow 1}} e^{-\frac{t}{\bar{\gamma}_{2 \rightarrow 1}}} d t-\frac{1}{\bar{\gamma}_{2 \rightarrow 1}} e^{-\frac{z}{\bar{\gamma}_{1 \rightarrow 1}}} \int_{0}^{\hat{\gamma}_{\mathrm{th}}} e^{-t\left(\frac{z}{\bar{\gamma}_{1 \rightarrow 1}}+\frac{1}{\bar{\gamma}_{2 \rightarrow 1}}\right)} d t \\
& +\left[1-e^{-\frac{z}{\bar{\gamma}_{1 \rightarrow 1}}}\right] e^{-\frac{\hat{\gamma}_{\text {th }}}{\bar{\gamma}_{2 \rightarrow 1}}} \\
& =\left.\left\{-e^{-\frac{t}{\bar{\gamma}_{2 \rightarrow 1}}}\right\}\right|_{0} ^{\hat{\gamma}_{\text {th }}}-\left.\frac{\bar{\gamma}_{1 \rightarrow 1}}{\left(\bar{\gamma}_{2 \rightarrow 1} z+\bar{\gamma}_{1 \rightarrow 1}\right)} e^{-\frac{z}{\bar{\gamma}_{1 \rightarrow 1}}}\left\{-e^{-t\left(\frac{z}{\bar{\gamma}_{1 \rightarrow 1}}+\frac{1}{\bar{\gamma}_{2 \rightarrow 1}}\right)}\right\}\right|_{0} ^{\hat{\gamma}_{\text {th }}} \\
& +\left[1-e^{-\frac{z}{\bar{\gamma}_{1 \rightarrow 1}}}\right] e^{-\frac{\hat{\gamma}_{\text {th }}}{\bar{\gamma}_{2 \rightarrow 1}}} \\
& =\left[1-e^{-\left(\frac{z}{\bar{\gamma}_{1 \rightarrow 1}}+\frac{\hat{\gamma}_{\text {th }}}{\bar{\gamma}_{2 \rightarrow 1}}\right)}\right]-\frac{\bar{\gamma}_{1 \rightarrow 1}}{\left(\bar{\gamma}_{2 \rightarrow 1} z+\bar{\gamma}_{1 \rightarrow 1}\right)} e^{-\frac{z}{\bar{\gamma}_{1 \rightarrow 1}}}\left[1-e^{-\hat{\gamma}_{\text {th }}\left(\frac{z}{\bar{\gamma}_{1 \rightarrow 1}}+\frac{1}{\bar{\gamma}_{2 \rightarrow 1}}\right)}\right] \text {. }
\end{aligned}
$$

\section{Partial channel phase information}

In presence of partial channel phase information, the stochastic behavior of RVs $X$ and $Y$ can be approximated as $\chi^{2}$ distributed with 4 and 2 degrees of freedom, respectively, i.e.,

$$
F_{X}(x)=1-\left(1+\frac{2 x}{\mathcal{G} \bar{\gamma}_{1 \rightarrow 1}}\right) e^{-\frac{2 x}{\mathcal{G} \bar{\gamma}_{1 \rightarrow 1}}} \quad x \geq 0, \quad f_{Y}(y)=\frac{1}{g \bar{\gamma}_{2 \rightarrow 1}} e^{-\frac{y}{g \bar{\gamma}_{2} \rightarrow 1}} \quad y \geq 0,
$$

where $\mathcal{G}$ and $g$ are known as the beamforming gains of the TBF scheme. Then, combining (37) with (40), it is possible to see that

$$
\begin{aligned}
& F_{Z}(z)=\int_{0}^{\hat{\gamma}_{\text {hh }}}\left\{1-\left[1+\frac{2 z(t+1)}{\mathcal{G} \bar{\gamma}_{1 \rightarrow 1}}\right] e^{-\frac{2 z(t+1)}{\mathcal{G} \bar{\gamma}_{1 \rightarrow 1}}}\right\} \frac{1}{g \bar{\gamma}_{2 \rightarrow 1}} e^{-\frac{t}{g \bar{\gamma}_{2 \rightarrow 1}}} d t \\
& +\left[1-\left(1+\frac{2 z}{\mathcal{G} \bar{\gamma}_{1 \rightarrow 1}}\right) e^{-\frac{2 z}{\mathcal{G} \bar{\gamma}_{1 \rightarrow 1}}}\right] e^{-\frac{\hat{\gamma}_{\text {th }}}{g \bar{\gamma}_{2 \rightarrow 1}}} \\
& =\int_{0}^{\hat{\gamma}_{\mathrm{th}}} \frac{1}{g \bar{\gamma}_{2 \rightarrow 1}} e^{-\frac{t}{g \bar{\gamma}_{2 \rightarrow 1}}} d t-\frac{1}{g \bar{\gamma}_{2 \rightarrow 1}}\left(1+\frac{2 z}{\mathcal{G} \bar{\gamma}_{1 \rightarrow 1}}\right) e^{-\frac{2 z}{\mathcal{G} \bar{\gamma}_{1 \rightarrow 1}}} \int_{0}^{\hat{\gamma}_{\mathrm{th}}} e^{-t\left(\frac{2 z}{\mathcal{G} \bar{\gamma}_{1 \rightarrow 1}}+\frac{1}{g \bar{\gamma}_{2 \rightarrow 1}}\right)} d t \\
& -\frac{1}{g \bar{\gamma}_{2 \rightarrow 1}}\left(\frac{2 z}{\mathcal{G} \bar{\gamma}_{1 \rightarrow 1}}\right) e^{-\frac{2 z}{\mathcal{G} \bar{\gamma}_{1 \rightarrow 1}}} \int_{0}^{\hat{\gamma}_{\text {th }}} t e^{-t\left(\frac{2 z}{\mathcal{G} \bar{\gamma}_{1 \rightarrow 1}}+\frac{1}{g \bar{\gamma}_{2 \rightarrow 1}}\right)} d t \\
& +\left[1-\left(1+\frac{2 z}{\mathcal{G} \bar{\gamma}_{1 \rightarrow 1}}\right) e^{-\frac{2 z}{\mathcal{G} \bar{\gamma}_{1 \rightarrow 1}}}\right] e^{-\frac{\hat{\gamma}_{\text {th }}}{g \bar{\gamma}_{2 \rightarrow 1}}} \\
& =\left.\left\{-e^{-\frac{t}{g \bar{\gamma}_{2 \rightarrow 1}}}\right\}\right|_{0} ^{\hat{\gamma}_{\text {th }}}-\left.\frac{2 z+\mathcal{G} \bar{\gamma}_{1 \rightarrow 1}}{2 g \bar{\gamma}_{2 \rightarrow 1} z+\mathcal{G} \bar{\gamma}_{1 \rightarrow 1}} e^{-\frac{2 z}{\mathcal{G} \bar{\gamma}_{1 \rightarrow 1}}}\left\{-e^{-t\left(\frac{2 z}{\overline{\mathcal{G}} \bar{\gamma}_{1 \rightarrow 1}}+\frac{1}{g \bar{\gamma}_{2 \rightarrow 1}}\right)}\right\}\right|_{0} ^{\hat{\gamma}_{\text {th }}} \\
& -\left.\frac{2 z}{2 g \bar{\gamma}_{2 \rightarrow 1} z+\mathcal{G} \bar{\gamma}_{1 \rightarrow 1}} e^{-\frac{2 z}{\mathcal{G} \bar{\gamma}_{1 \rightarrow 1}}}\left\{-e^{-t\left(\frac{2 z}{\mathcal{G} \bar{\gamma}_{1 \rightarrow 1}}+\frac{1}{g \bar{\gamma}_{2 \rightarrow 1}}\right)}\left[t+\frac{1}{\left(\frac{2 z}{\mathcal{G} \bar{\gamma}_{1 \rightarrow 1}}+\frac{1}{g \bar{\gamma}_{2 \rightarrow 1}}\right)}\right]\right\}\right|_{0} ^{\hat{\gamma}_{\text {th }}} \\
& +\left[1-\left(1+\frac{2 z}{\mathcal{G} \bar{\gamma}_{1 \rightarrow 1}}\right) e^{-\frac{2 z}{\mathcal{G} \bar{\gamma}_{1 \rightarrow 1}}}\right] e^{-\frac{\hat{\gamma}_{\text {th }}}{g \bar{\gamma}_{2 \rightarrow 1}}} \\
& =1-\left(1+\frac{2 z}{\mathcal{G} \bar{\gamma}_{1 \rightarrow 1}}\right) e^{-\left(\frac{2 z}{\mathcal{G} \bar{\gamma}_{1 \rightarrow 1}}+\frac{\hat{\gamma}_{\text {th }}}{g \bar{\gamma}_{2 \rightarrow 1}}\right)}+\frac{2 z \hat{\gamma}_{\text {th }}}{2 g \bar{\gamma}_{2 \rightarrow 1} z+\mathcal{G} \bar{\gamma}_{1 \rightarrow 1}} e^{-\left[\frac{2 z\left(\hat{\gamma}_{\text {th }}+1\right)}{\mathcal{G} \bar{\gamma}_{1 \rightarrow 1}}+\frac{\hat{\gamma}_{\text {th }}}{g \bar{\gamma}_{2 \rightarrow 1}}\right]} \\
& -\left[\frac{2 z+\mathcal{G} \bar{\gamma}_{1 \rightarrow 1}}{2 g \bar{\gamma}_{2 \rightarrow 1} z+\mathcal{G} \bar{\gamma}_{1 \rightarrow 1}}+\frac{2 z g \mathcal{G} \bar{\gamma}_{1 \rightarrow 1} \bar{\gamma}_{2 \rightarrow 1}}{\left(2 g \bar{\gamma}_{2 \rightarrow 1} z+\mathcal{G} \bar{\gamma}_{1 \rightarrow 1}\right)^{2}}\right] e^{-\frac{2 z}{\overline{\mathcal{G}} \bar{\gamma}_{1 \rightarrow 1}}}\left[1-e^{-\hat{\gamma_{\text {th }}}\left(\frac{2 z}{\mathcal{G} \bar{\gamma}_{1 \rightarrow 1}}+\frac{1}{g \bar{\gamma}_{2 \rightarrow 1}}\right)}\right] .
\end{aligned}
$$




\section{Full channel phase information}

In presence of full channel phase information, the stochastic behavior of RVs $X$ and $Y$ can be approximated as $\chi^{2}$ distributed RVs with 4 and 1 degrees of freedom, respectively, i.e.,

$$
F_{X}(x)=1-\left(1+\frac{2 x}{\mathcal{G} \bar{\gamma}_{1 \rightarrow 1}}\right) e^{-\frac{2 x}{\mathcal{G} \bar{\gamma}_{1 \rightarrow 1}}} \quad x \geq 0, \quad f_{Y}(y)=\frac{1}{\sqrt{2 \pi g \bar{\gamma}_{2 \rightarrow 1} y}} e^{-\frac{y}{2 g \bar{\gamma}_{2} \rightarrow 1}} \quad y \geq 0,
$$

where the CDF of the RV $Y$ is given by

$$
F_{Y}(y)=\operatorname{erf}\left(\sqrt{\frac{y}{2 g \bar{\gamma}_{2 \rightarrow 1}}}\right) \quad y \geq 0 .
$$

Combining (37) with (42) and (43), it is possible to see that

$$
\begin{aligned}
F_{Z}(z)= & \int_{0}^{\hat{\gamma}_{\mathrm{th}}}\left\{1-\left[1+\frac{2 z(t+1)}{\mathcal{G} \bar{\gamma}_{1 \rightarrow 1}}\right] e^{-\frac{2 z(t+1)}{\mathcal{G} \bar{\gamma}_{1 \rightarrow 1}}}\right\} \frac{1}{\sqrt{2 \pi g \bar{\gamma}_{2 \rightarrow 1} t}} e^{-\frac{t}{2 g \bar{\gamma}_{2 \rightarrow 1}}} d t \\
& +\left[1-\left(1+\frac{2 z}{\mathcal{G} \bar{\gamma}_{1 \rightarrow 1}}\right) e^{-\frac{2 z}{\mathcal{G} \bar{\gamma}_{1 \rightarrow 1}}}\right]\left[1-\operatorname{erf}\left(\sqrt{\frac{\hat{\gamma}_{\mathrm{th}}}{2 g \bar{\gamma}_{2 \rightarrow 1}}}\right)\right] \\
= & \frac{1}{\sqrt{2 \pi g \bar{\gamma}_{2 \rightarrow 1}}} \int_{0}^{\hat{\gamma} \mathrm{th}} \frac{1}{\sqrt{t}} e^{-\frac{t}{2 g \bar{\gamma}_{2 \rightarrow 1}}} d t \\
& -\frac{1}{\sqrt{2 \pi g \bar{\gamma}_{2 \rightarrow 1}}} e^{-\frac{2 z}{\mathcal{G} \bar{\gamma}_{1 \rightarrow 1}}}\left(1+\frac{2 z}{\mathcal{G} \bar{\gamma}_{1 \rightarrow 1}}\right) \int_{0}^{\hat{\gamma_{\mathrm{th}}}} \frac{1}{\sqrt{t}} e^{-t\left(\frac{1}{2 g \bar{\gamma}_{2 \rightarrow 1}}+\frac{2 z}{\mathcal{G} \bar{\gamma}_{1 \rightarrow 1}}\right)} d t \\
& -\frac{1}{\sqrt{2 \pi g \bar{\gamma}_{2 \rightarrow 1}}} e^{-\frac{2 z}{\mathcal{G} \bar{\gamma}_{1 \rightarrow 1}}}\left(\frac{2 z}{\mathcal{G} \bar{\gamma}_{1 \rightarrow 1}}\right) \int_{0}^{\hat{\gamma} \text { th }} \sqrt{t} e^{-t\left(\frac{1}{2 g \bar{\gamma}_{2 \rightarrow 1}}+\frac{2 z}{\mathcal{G} \bar{\gamma}_{1 \rightarrow 1}}\right)} d t \\
& +\left[1-\left(1+\frac{2 z}{\mathcal{G} \bar{\gamma}_{1 \rightarrow 1}}\right) e^{-\frac{2 z}{\mathcal{G} \bar{\gamma}_{1 \rightarrow 1}}}\right]\left[1-\operatorname{erf}\left(\sqrt{\frac{\hat{\gamma}_{\text {th }}}{2 g \bar{\gamma}_{2 \rightarrow 1}}}\right)\right] .
\end{aligned}
$$

At this stage of the analysis, we use the definite integral expressions

$$
\int_{0}^{u} \sqrt{x} e^{-\alpha x} d x=-\frac{\sqrt{u}}{\alpha} e^{-\alpha u}+\frac{\sqrt{\pi}}{2 \alpha^{\frac{3}{2}}} \operatorname{erf}(\sqrt{\alpha u}), \quad \int_{0}^{u} \frac{1}{\sqrt{x}} e^{-\alpha x} d x=\sqrt{\frac{\pi}{\alpha}} \operatorname{erf}(\sqrt{\alpha u}),
$$

to finally obtain the following closed-form formula:

$$
\begin{aligned}
& F_{Z}(z)=1-\left(1+\frac{2 z}{\mathcal{G} \bar{\gamma}_{1 \rightarrow 1}}\right)\left(\frac{1}{2 g \bar{\gamma}_{2 \rightarrow 1}}\right)^{\frac{1}{2}}\left(\frac{1}{2 g \bar{\gamma}_{2 \rightarrow 1}}+\frac{2 z}{\mathcal{G} \bar{\gamma}_{1 \rightarrow 1}}\right)^{-\frac{1}{2}} \\
& e^{-\frac{2 z}{\mathcal{G} \bar{\gamma}_{1 \rightarrow 1}}} \operatorname{erf}\left(\sqrt{\left(\frac{1}{2 g \bar{\gamma}_{2 \rightarrow 1}}+\frac{2 z}{\mathcal{G} \bar{\gamma}_{1 \rightarrow 1}}\right) \hat{\gamma}_{\text {th }}}\right) \\
& -\frac{2 z}{\mathcal{G} \bar{\gamma}_{1 \rightarrow 1}}\left(\frac{1}{2 \pi g \bar{\gamma}_{2 \rightarrow 1}}\right)^{\frac{1}{2}} e^{-\frac{2 z}{\mathcal{G} \bar{\gamma}_{1 \rightarrow 1}}}\left\{\frac{\sqrt{\pi}}{2}\left(\frac{1}{2 g \bar{\gamma}_{2 \rightarrow 1}}+\frac{2 z}{\mathcal{G} \bar{\gamma}_{1 \rightarrow 1}}\right)^{-\frac{3}{2}}\right. \\
& \operatorname{erf}\left(\sqrt{\left(\frac{1}{2 g \bar{\gamma}_{2 \rightarrow 1}}+\frac{2 z}{\mathcal{G} \bar{\gamma}_{1 \rightarrow 1}}\right) \hat{\gamma}_{\text {th }}}\right)-\sqrt{\hat{\gamma}_{\text {th }}}\left(\frac{1}{2 g \bar{\gamma}_{2 \rightarrow 1}}+\frac{2 z}{\mathcal{G} \bar{\gamma}_{1 \rightarrow 1}}\right)^{-1} \\
& \left.e^{-\left(\frac{1}{2 g \bar{\gamma}_{2} \rightarrow 1}+\frac{2 z}{\mathcal{G} \bar{\gamma}_{1 \rightarrow 1}}\right) \hat{\gamma}_{\mathrm{th}}}\right\}-\left(1+\frac{2 z}{\mathcal{G} \bar{\gamma}_{1 \rightarrow 1}}\right) e^{-\frac{2 z}{\mathcal{G} \bar{\gamma}_{1} \rightarrow 1}} \operatorname{erfc}\left(\sqrt{\frac{\hat{\gamma}_{\mathrm{th}}}{2 g \bar{\gamma}_{2 \rightarrow 1}}}\right) \text {. }
\end{aligned}
$$




\section{Appendix 2}

\section{Scheduling of silence periods based on direct-link signal strength}

When TBF is combined with the scheduling of SPs based on the strength of the directlink, see (10), the CDF of the received SINR becomes

$$
F_{Z}(z)=F_{Z}\left(z \mid X>\check{\gamma}_{\text {th }}\right) \operatorname{Pr}\left\{X>\check{\gamma}_{\text {th }}\right\}+F_{X}\left(z \mid X \leq \check{\gamma}_{\text {th }}\right) \operatorname{Pr}\left\{X \leq \check{\gamma}_{\text {th }}\right\} \quad z \geq 0,
$$

where the different probabilistic models that are used to approximate the distributions of RVs $X$ and $Y$ will depend on the specific study case. In this situation, $\check{\gamma}_{\text {th }}$ represents the threshold that defines the SR condition in the direct-link.

\section{Single-antenna system (no channel phase information)}

In absence of channel phase information, the distributions for RVs $X$ and $Y$ were reported in (38), and the conditional distributions for RV $X$ are given by

$$
\begin{aligned}
& F_{X}\left(x \mid X \leq \check{\gamma}_{\text {th }}\right)=\frac{1-e^{-\frac{x}{\bar{\gamma}_{1 \rightarrow 1}}}}{1-e^{-\frac{\check{\gamma}_{\text {th }}}{\bar{\gamma}_{1 \rightarrow 1}}}} \quad 0 \leq x \leq \check{\gamma}_{\text {th }}, \\
& F_{X}\left(x \mid X>\check{\gamma}_{\text {th }}\right)=1-e^{-\frac{\left(x-\check{\gamma}_{\text {th }}\right)}{\bar{\gamma}_{1 \rightarrow 1}}} \quad x>\check{\gamma}_{\text {th }} .
\end{aligned}
$$

Then, combining (47) with (48), we get

$$
\begin{aligned}
& F_{Z}(z)=\operatorname{Pr}\left\{X>\check{\gamma}_{\text {th }}\right\} \int_{0}^{+\infty} F_{X}\left(z(t+1) \mid X>\check{\gamma}_{\text {th }}\right) f_{Y}(t) d t+F_{X}\left(z \mid X \leq \check{\gamma}_{\text {th }}\right) \operatorname{Pr}\left\{X \leq \check{\gamma}_{\text {th }}\right\} \\
& =e^{-\frac{\check{\gamma}_{\text {th }}}{\bar{\gamma}_{1 \rightarrow 1}}} \int_{\max \left\{\frac{\check{\gamma}_{\text {th }}}{z}-1,0\right\}}^{+\infty}\left[1-e^{-\frac{\left[z(t+1)-\check{\gamma}_{\text {th }}\right]}{\bar{\gamma}_{1 \rightarrow 1}}}\right]\left[\frac{1}{\bar{\gamma}_{2 \rightarrow 1}} e^{-\frac{t}{\bar{\gamma}_{2 \rightarrow 1}}}\right] d t+1-e^{-\frac{\min \left\{z, \check{\gamma}_{\text {th }}\right\}}{\bar{\gamma}_{1 \rightarrow 1}}} \\
& =\left.e^{-\frac{\check{\gamma}_{\text {th }}}{\bar{\gamma}_{1 \rightarrow 1}}}\left\{-e^{-\frac{t}{\bar{\gamma}_{2 \rightarrow 1}}}\right\}\right|_{\max \left\{\frac{\check{\gamma}_{\text {th }}}{z}-1,0\right\}} ^{+\infty}+1-e^{-\frac{\min \left\{z, \check{\gamma}_{\text {th }}\right\}}{\bar{\gamma}_{1 \rightarrow 1}}} \\
& -\left.\frac{1}{\bar{\gamma}_{2 \rightarrow 1}} e^{-\frac{z}{\bar{\gamma}_{1 \rightarrow 1}}}\left(\frac{z}{\bar{\gamma}_{1 \rightarrow 1}}+\frac{1}{\bar{\gamma}_{2 \rightarrow 1}}\right)^{-1}\left\{-e^{-t\left(\frac{z}{\bar{\gamma}_{1 \rightarrow 1}}+\frac{1}{\bar{\gamma}_{2 \rightarrow 1}}\right)}\right\}\right|_{\max \left\{\frac{\check{\gamma}_{\text {th }}}{z}-1,0\right\}} ^{+\infty} \\
& =e^{-\frac{\check{\gamma}_{\text {th }}}{\bar{\gamma}_{1 \rightarrow 1}}} e^{-\frac{\max \left\{\frac{\check{\gamma}_{\text {th }}}{z}-1,0\right\}}{\bar{\gamma}_{2 \rightarrow 1}}}+1-e^{-\frac{\min \left\{z, \check{\gamma}_{\text {th }}\right\}}{\bar{\gamma}_{1 \rightarrow 1}}} \\
& -e^{-\frac{z}{\bar{\gamma}_{1 \rightarrow 1}}}\left(\frac{\bar{\gamma}_{1 \rightarrow 1}}{z \bar{\gamma}_{2 \rightarrow 1}+\bar{\gamma}_{1 \rightarrow 1}}\right) e^{-\left(\frac{z}{\bar{\gamma}_{1 \rightarrow 1}}+\frac{1}{\bar{\gamma}_{2 \rightarrow 1}}\right) \max \left\{\frac{\check{\gamma}_{\text {th }}}{z}-1,0\right\}} .
\end{aligned}
$$

\section{Partial channel phase information}

In presence of partial channel phase information, the stochastic behavior of RVs $X$ and $Y$ was reported in (40), and the conditional distributions for RV $X$ are given by

$$
\begin{aligned}
& F_{X}\left(x \mid X \leq \check{\gamma}_{\text {th }}\right)=\frac{1-\left(1+\frac{2 x}{\mathcal{G} \bar{\gamma}_{1 \rightarrow 1}}\right) e^{-\frac{2 x}{\mathcal{G} \bar{\gamma}_{1 \rightarrow 1}}}}{1-\left(1+\frac{2 \check{\gamma}_{\text {th }}}{\mathcal{G} \bar{\gamma}_{1 \rightarrow 1}}\right) e^{-\frac{2 \check{\gamma}_{\text {th }}}{\mathcal{G} \bar{\gamma}_{1 \rightarrow 1}}}} \quad 0 \leq x \leq \check{\gamma}_{\text {th }}, \\
& F_{X}\left(x \mid X>\check{\gamma}_{\mathrm{th}}\right)=1-\frac{\left(1+\frac{2 x}{\mathcal{G} \bar{\gamma}_{1 \rightarrow 1}}\right) e^{-\frac{2 x}{\mathcal{G} \bar{\gamma}_{1 \rightarrow 1}}}}{\left(1+\frac{2 \check{\gamma}_{\mathrm{th}}}{\mathcal{G} \bar{\gamma}_{1 \rightarrow 1}}\right) e^{-\frac{2 \check{\gamma}_{\mathrm{th}}}{\mathcal{G} \bar{\gamma}_{1 \rightarrow 1}}}} x>\check{\gamma}_{\mathrm{th}} .
\end{aligned}
$$

Then, combining (47) with (50) and (51), it is found that 


$$
\begin{aligned}
& F_{Z}(z)=\operatorname{Pr}\left\{X>\check{\gamma}_{\text {th }}\right\} \int_{0}^{+\infty} F_{X}\left(z(t+1) \mid X>\check{\gamma}_{\text {th }}\right) f_{Y}(t) d t+F_{X}\left(z \mid X \leq \check{\gamma}_{\text {th }}\right) \operatorname{Pr}\left\{X \leq \check{\gamma}_{\text {th }}\right\} \\
& =\int_{\max \left\{\frac{\check{\gamma}_{\text {th }}}{z}-1,0\right\}}^{+\infty}\left[\left(1+\frac{2 \check{\gamma}_{\text {th }}}{\mathcal{G} \bar{\gamma}_{1 \rightarrow 1}}\right) e^{-\frac{2 \check{\gamma}_{\text {th }}}{\mathcal{G} \bar{\gamma}_{1 \rightarrow 1}}}-\left(1+\frac{2 z(t+1)}{\mathcal{G} \bar{\gamma}_{1 \rightarrow 1}}\right) e^{-\frac{2 z(t+1)}{\mathcal{G} \bar{\gamma}_{1 \rightarrow 1}}}\right] \\
& \times\left[\frac{1}{g \bar{\gamma}_{2 \rightarrow 1}} e^{-\frac{t}{g \bar{\gamma}_{2 \rightarrow 1}}}\right] d t+1-\left(1+\frac{2 \min \left\{z, \check{\gamma}_{\text {th }}\right\}}{\mathcal{G} \bar{\gamma}_{1 \rightarrow 1}}\right) e^{-\frac{2 \min \left\{z, \check{\gamma}_{\text {th }}\right\}}{\mathcal{G} \bar{\gamma}_{1 \rightarrow 1}}} \\
& =\left.\left(1+\frac{2 \check{\gamma}_{\text {th }}}{\mathcal{G} \bar{\gamma}_{1 \rightarrow 1}}\right) e^{-\frac{2 \check{\gamma}_{\text {th }}}{\mathcal{G}}}\left\{-e^{-\frac{t}{g \bar{\gamma}_{2 \rightarrow 1}}}\right\}\right|_{\max \left\{\frac{\check{\gamma}_{\text {th }}}{z}-1,0\right\}} ^{+\infty} \\
& +1-\left(1+\frac{2 \min \left\{z, \check{\gamma}_{\text {th }}\right\}}{\mathcal{G} \bar{\gamma}_{1 \rightarrow 1}}\right) e^{-\frac{2 \min \left\{z, \check{\gamma}_{\text {th }}\right\}}{\mathcal{G} \bar{\gamma}_{1 \rightarrow 1}}} \\
& -\left(1+\frac{2 z}{\mathcal{G} \bar{\gamma}_{1 \rightarrow 1}}\right)\left(\frac{1}{g \bar{\gamma}_{2 \rightarrow 1}}\right)\left(\frac{2 z}{\mathcal{G} \bar{\gamma}_{1 \rightarrow 1}}+\frac{1}{g \bar{\gamma}_{2 \rightarrow 1}}\right)^{-1} \\
& \left.e^{-\frac{2 z}{\mathcal{G} \bar{\gamma}_{1 \rightarrow 1}}}\left\{-e^{-t\left(\frac{2 z}{\mathcal{G} \bar{\gamma}_{1 \rightarrow 1}}+\frac{1}{g \bar{\gamma}_{2} \rightarrow 1}\right)}\right\}\right|_{\max \left\{\frac{\check{\gamma}_{\text {th }}}{z}-1,0\right\}} ^{+\infty} \\
& -\left(\frac{2 z}{\mathcal{G} \bar{\gamma}_{1 \rightarrow 1}}\right)\left(\frac{1}{g \bar{\gamma}_{2 \rightarrow 1}}\right)\left(\frac{2 z}{\mathcal{G} \bar{\gamma}_{1 \rightarrow 1}}+\frac{1}{g \bar{\gamma}_{2 \rightarrow 1}}\right)^{-2} \\
& \left.e^{-\frac{2 z}{\mathcal{G} \bar{\gamma}_{1 \rightarrow 1}}}\left\{-e^{-t\left(\frac{2 z}{\mathcal{G} \bar{\gamma}_{1 \rightarrow 1}}+\frac{1}{g \bar{\gamma}_{2 \rightarrow 1}}\right)}\left[\left(\frac{2 z}{\mathcal{G} \bar{\gamma}_{1 \rightarrow 1}}+\frac{1}{g \bar{\gamma}_{2 \rightarrow 1}}\right) t+1\right]\right\}\right|_{\max } ^{+\infty}\left\{\frac{\check{y}_{\text {th }}}{z}-1,0\right\} \\
& =\left(1+\frac{2 \check{\gamma}_{\text {th }}}{\mathcal{G} \bar{\gamma}_{1 \rightarrow 1}}\right) e^{-\frac{2 \check{\gamma}_{\text {th }}}{\mathcal{G} \bar{\gamma}_{1 \rightarrow 1}}} e^{-\frac{\max \left\{\frac{\check{y}_{\text {th }}}{z}-1,0\right\}}{g \bar{\gamma}_{2 \rightarrow 1}}}+1-\left(1+\frac{2 \min \left\{z, \check{\gamma}_{\text {th }}\right\}}{\mathcal{G} \bar{\gamma}_{1 \rightarrow 1}}\right) e^{-\frac{2 \min \left\{z, \check{\mathrm{r}}_{\mathrm{th}}\right\}}{\mathcal{G} \bar{\gamma}_{1 \rightarrow 1}}} \\
& -\left(1+\frac{2 z}{\mathcal{G} \bar{\gamma}_{1 \rightarrow 1}}\right)\left(\frac{1}{g \bar{\gamma}_{2 \rightarrow 1}}\right)\left(\frac{2 z}{\mathcal{G} \bar{\gamma}_{1 \rightarrow 1}}+\frac{1}{g \bar{\gamma}_{2 \rightarrow 1}}\right)^{-1} e^{-\frac{2 z}{\mathcal{G} \bar{\gamma}_{1 \rightarrow 1}}} \\
& e^{-\max \left\{\frac{\check{\gamma}_{\text {th }}}{z}-1,0\right\}\left(\frac{2 z}{\mathcal{G} \bar{\gamma}_{1 \rightarrow 1}}+\frac{1}{g \bar{\gamma}_{2 \rightarrow 1}}\right)}-\left(\frac{2 z}{\mathcal{G} \bar{\gamma}_{1 \rightarrow 1}}\right)\left(\frac{1}{g \bar{\gamma}_{2 \rightarrow 1}}\right)\left(\frac{2 z}{\mathcal{G} \bar{\gamma}_{1 \rightarrow 1}}+\frac{1}{g \bar{\gamma}_{2 \rightarrow 1}}\right)^{-2} \\
& e^{-\frac{2 z}{\mathcal{G} \bar{\gamma}_{1 \rightarrow 1}}} e^{-\max \left\{\frac{\check{y}_{\text {th }}}{z}-1,0\right\}\left(\frac{2 z}{\mathcal{G} \bar{\gamma}_{1 \rightarrow 1}}+\frac{1}{g \bar{\gamma}_{2 \rightarrow 1}}\right)}\left[\max \left\{\frac{\check{\gamma}_{\text {th }}}{z}-1,0\right\}\left(\frac{2 z}{\mathcal{G} \bar{\gamma}_{1 \rightarrow 1}}+\frac{1}{g \bar{\gamma}_{2 \rightarrow 1}}\right)+1\right] \text {. }
\end{aligned}
$$

\section{Full channel phase information}

In presence of full channel phase information, the stochastic behavior of RVs $X$ and $Y$ was reported in (42) and (43), and the conditional distributions for RV $X$ attend the form given in (50) and (51). Then, combining (47) with (50) and (51), it is possible to observe that

$$
\begin{aligned}
& F_{Z}(z)=\operatorname{Pr}\left\{X>\check{\gamma}_{\text {th }}\right\} \int_{0}^{+\infty} F_{X}\left(z(t+1) \mid X>\check{\gamma}_{\text {th }}\right) f_{Y}(t) d t+F_{X}\left(z \mid X \leq \check{\gamma}_{\text {th }}\right) \operatorname{Pr}\left\{X \leq \check{\gamma}_{\text {th }}\right\} \\
& =\int_{\max \left\{\frac{\check{\gamma}_{\text {th }}}{z}-1,0\right\}}^{+\infty}\left[\left(1+\frac{2 \check{\gamma}_{\text {th }}}{\mathcal{G} \bar{\gamma}_{1 \rightarrow 1}}\right) e^{-\frac{2 \check{\gamma}_{\text {th }}}{\mathcal{G} \bar{\gamma}_{1 \rightarrow 1}}}-\left(1+\frac{2 z(t+1)}{\mathcal{G} \bar{\gamma}_{1 \rightarrow 1}}\right) e^{-\frac{2 z(t+1)}{\mathcal{G} \bar{\gamma}_{1 \rightarrow 1}}}\right] \\
& \times\left[\frac{1}{\sqrt{2 \pi g \bar{\gamma}_{2 \rightarrow 1} t}} e^{-\frac{t}{2 g \bar{\gamma}_{2} \rightarrow 1}}\right] d t+1-\left(1+\frac{2 \min \left\{z, \check{\gamma}_{\mathrm{th}}\right\}}{\mathcal{G} \bar{\gamma}_{1 \rightarrow 1}}\right) e^{-\frac{2 \min \left\{z, \check{\gamma}_{\mathrm{th}}\right\}}{\mathcal{G} \bar{\gamma}_{1 \rightarrow 1}}} \\
& =\left(1+\frac{2 \check{\gamma}_{\text {th }}}{\mathcal{G} \bar{\gamma}_{1 \rightarrow 1}}\right) e^{-\frac{2 \check{\gamma}_{\text {th }}}{\mathcal{G} \bar{\gamma}_{1 \rightarrow 1}}} \int_{\max \left\{\frac{\check{\gamma}_{\text {th }}}{z}-1,0\right\}}^{+\infty} \frac{1}{\sqrt{2 \pi g \bar{\gamma}_{2 \rightarrow 1} t}} e^{-\frac{t}{2 g \bar{\gamma}_{2 \rightarrow 1}}} d t \\
& -\left(1+\frac{2 z}{\mathcal{G} \bar{\gamma}_{1 \rightarrow 1}}\right) \frac{1}{\sqrt{2 \pi g \bar{\gamma}_{2 \rightarrow 1}}} e^{-\frac{2 z}{\mathcal{G} \bar{\gamma}_{1 \rightarrow 1}}} \int_{\max \left\{\frac{\check{\gamma}_{h h}}{z}-1,0\right\}}^{+\infty} \frac{1}{\sqrt{t}} e^{-t\left(\frac{2 z}{\mathcal{G} \bar{\gamma}_{1 \rightarrow 1}}+\frac{1}{2 g \bar{\gamma}_{2 \rightarrow 1}}\right)} d t \\
& -\left(\frac{2 z}{\mathcal{G} \bar{\gamma}_{1 \rightarrow 1}}\right) \frac{1}{\sqrt{2 \pi g \bar{\gamma}_{2 \rightarrow 1}}} e^{-\frac{2 z}{\mathcal{G} \bar{\gamma}_{1 \rightarrow 1}}} \int_{\max \left\{\frac{\check{\gamma}_{\text {th }}}{z}-1,0\right\}}^{+\infty} \sqrt{t} e^{-t\left(\frac{2 z}{\overline{\mathcal{G}} \bar{\gamma}_{1 \rightarrow 1}}+\frac{1}{2 g \bar{\gamma}_{2 \rightarrow 1}}\right)} d t \\
& +1-\left(1+\frac{2 \min \left\{z, \check{\gamma}_{\text {th }}\right\}}{\mathcal{G} \bar{\gamma}_{1 \rightarrow 1}}\right) e^{-\frac{2 \min \left\{z, \check{\gamma}_{\text {th }}\right\}}{\mathcal{G} \bar{\gamma}_{1 \rightarrow 1}}} .
\end{aligned}
$$


At this stage of the analysis, we use the definite integral expressions

$$
\int_{u}^{+\infty} \sqrt{x} e^{-\alpha x} d x=\frac{\sqrt{u}}{\alpha} e^{-\alpha u}+\frac{\sqrt{\pi}}{2 \alpha^{\frac{3}{2}}} \operatorname{erfc}(\sqrt{\alpha u}), \quad \int_{u}^{+\infty} \frac{1}{\sqrt{x}} e^{-\alpha x} d x=\sqrt{\frac{\pi}{\alpha}} \operatorname{erfc}(\sqrt{\alpha u}),
$$

to obtain the following closed-form formula:

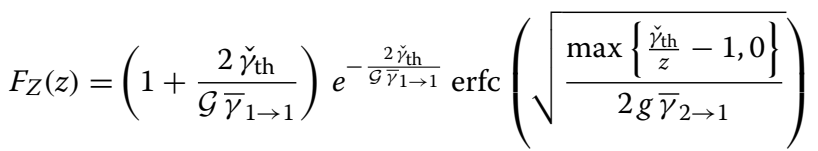

$$
\begin{aligned}
& -\left(1+\frac{2 z}{\mathcal{G} \bar{\gamma}_{1 \rightarrow 1}}\right) \sqrt{\frac{\mathcal{G} \bar{\gamma}_{1 \rightarrow 1}}{4 z g \bar{\gamma}_{2 \rightarrow 1}+\mathcal{G} \bar{\gamma}_{1 \rightarrow 1}}} e^{-\frac{2 z}{\mathcal{G} \bar{\gamma}_{1 \rightarrow 1}}} \\
& \operatorname{erfc}\left(\sqrt{\max \left\{\frac{\check{\gamma}_{\text {th }}}{z}-1,0\right\}\left(\frac{2 z}{\mathcal{G} \bar{\gamma}_{1 \rightarrow 1}}+\frac{1}{2 g \bar{\gamma}_{2 \rightarrow 1}}\right)}\right) \\
& -\left(\frac{2 z}{\mathcal{G} \bar{\gamma}_{1 \rightarrow 1}}\right) \frac{1}{\sqrt{2 \pi g \bar{\gamma}_{2 \rightarrow 1}}} e^{-\frac{2 z}{\mathcal{G} \bar{\gamma}_{1 \rightarrow 1}}}\left\{\frac{\sqrt{\max \left\{\frac{\check{\gamma}_{\text {th }}}{z}-1,0\right\}}}{\left(\frac{2 z}{\mathcal{G} \bar{\gamma}_{1 \rightarrow 1}}+\frac{1}{2 g \bar{\gamma}_{2 \rightarrow 1}}\right)} e^{-\max \left\{\frac{\check{\gamma}_{\text {h }}}{z}-1,0\right\}\left(\frac{2 z}{\mathcal{G} \bar{\gamma}_{1 \rightarrow 1}}+\frac{1}{2 g \bar{\gamma}_{2 \rightarrow 1}}\right)}\right. \\
& \left.+\frac{\sqrt{\pi}}{2\left(\frac{2 z}{\mathcal{G} \bar{\gamma}_{1 \rightarrow 1}}+\frac{1}{2 g \bar{\gamma}_{2 \rightarrow 1}}\right)^{\frac{3}{2}}} \operatorname{erfc}\left[\sqrt{\max \left\{\frac{\check{\gamma}_{\text {th }}}{z}-1,0\right\}\left(\frac{2 z}{\mathcal{G} \bar{\gamma}_{1 \rightarrow 1}}+\frac{1}{2 g \bar{\gamma}_{2 \rightarrow 1}}\right)}\right]\right\} \\
& +1-\left(1+\frac{2 \min \left\{z, \check{\gamma}_{\text {th }}\right\}}{\mathcal{G} \bar{\gamma}_{1 \rightarrow 1}}\right) e^{-\frac{2 \min \left\{z, \check{\gamma}_{\text {th }}\right\}}{\mathcal{G} \bar{\gamma}_{1 \rightarrow 1}}} \text {. }
\end{aligned}
$$

\section{Competing interests}

The authors declare that they have no competing interests.

\section{Acknowledgements}

This study was prepared in Spectrum Management for Future Wireless Systems (SMAS) and Interference Management for Wireless Networks Beyond Present Horizon (IMANET) project frameworks, and was supported in part by the Academy of Finland (under grant 133652), the Finnish Funding Agency for Technology and Innovation (Tekes), Nokia Siemens Networks, Ericsson Finland, Nokia and Elektrobit Ltd. This article was presented in part at the Future Network \& Mobile Summit (FutureNetw) 2011: Workshop on Broadband Femtocell Networks [26].

\section{Author details}

${ }^{1}$ Department of Communications and Networking, Aalto University, P.O. Box 13000, Fl-00076 Aalto, Finland. ${ }^{2}$ Ericsson R\&D Center, Elektroniikkatie 10, FI-90590, Oulu, Finland.

Received: 22 November 2011 Accepted: 24 August 2012

Published: 18 September 2012

\section{References}

1. MI Silventoinen, M Kuusela, PA Ranta, in Proc. IEEE Int. Conf. on Universal Personal Commun., Analysis of a new channel access method for home base station. vol. 2 (Cambridge, Massachusetts, USA, 1996), pp. 930-935

2. MI Silventoinen, M Kuusela, PA Ranta, in Proc. IEEE Int. Conf. Personal Wireless Commun, Total frequency hopping for home base stations. (New Delhi, India, 1996), pp. 257-261

3. JN Latta, The WAVE Report, Issue \# 9034: Alcatel readies home base station (1999), http://www.wave-report.com/ 1999\%20Wave\%20issues/wave9034.html

4. D Chambers, ThinkFemtocell: femtocell history (2008), http://www.thinkfemtocell.com/FAQs/femtocell-history.html

5. V Chandrasekhar, J Andrews, A Gatherer, Femtocell networks: a survey. IEEE Commun. Mag. 46(9), 59-67 (2008)

6. H Claussen, in Proc. IEEE Int. Symp. on Personal, Indoor and Mobile Radio Commun, Performance of macro- and co-channel femtocells in a hierarchical cell structure. (Athens, Greece, 2007), pp. 1-5

7. HA Mahmoud, I Guvenc, in Proc. IEEE Int. Symp. on Personal, Indoor and Mobile Radio Commun, A comparative study of different deployment modes for femtocell networks. (Tokyo, Japan, 2009), pp. 1-5

8. HC Lee, DC Oh, YH Lee, in Proc. Int. Conf. on Commun, Mitigation of inter-femtocell interference with adaptive fractional frequency reuse. (Cape Town, South Africa, 2010), pp. 1-5

9. MZ Chowdhury, YM Jang, ZJ Haas, in Proc. Int. Conf. on Ubiquitous and Future Networks, Interference mitigation using dynamic frequency re-use for dense femtocell network architectures. (Jeju Island, South Korea, 2010), pp. 256-261

10. X Li, L Qian, D Kataria, in Proc. Annual Conf. on Inform. Sciences and Systems, Downlink power control in co-channel macrocell femtocell overlay. (Baltimore, Maryland, USA, 2009), pp. 383-388 
11. HS Jo, C Mun, J Moon, JG Yook, Interference mitigation using uplink power control for two-tier femtocell networks. IEEE Trans. Wirel. Commun. 8(10), 4906-4910 (2009)

12. S Park, W Seo, S Choi, D Hong, A beamforming codebook restriction for cross-tier interference coordination in two-tier femtocell networks. IEEE Trans. Veh. Tech. 60(4), 1651-1663 (2011)

13. M Husso, J Hämäläinen, R Jäntti, J Li, E Mutafungwa, R Wichman, Z Zheng, AM Wyglinski, Interference mitigation by practical transmit beamforming methods in closed femtocells. EURASIP J. Wirel. Commun. Network. 2010, 1-12 (2010)

14. M Husso, Z Zheng, J Hämäläinen, E Mutafungwa, in Proc. IEEE Int. Symp. on Personal, Indoor and Mobile Radio Commun. Workhops, Dominant interferer mitigation in closed femtocell deployment. (Istambul, Turkey, 2010), pp. 169-174

15. B Ghimire, G Auer, H Haas, Busy burst enabled coordinated multipoint network with decentralized control. IEEE Trans. Wirel. Commun. 10(10), 3310-3320 (2011)

16. Z Zheng, J Hämäläinen, Y Yang, in Proc. Int. Workshop on Multi-Carrier Systems and Solutions, Practical resource scheduling and power control optimization for LTE femtocell networks. (Herrsching, Germany, 2011), pp. 1-5

17. HC Lee, DC Oh, YH Lee, in Proc. IEEE Int. Conf. on Commun, Coordinated user scheduling with transmit beamforming in the presence of inter-femtocell interference. (Kyoto, Japan, 2011), pp. 1-5

18. J Giese, MA Amin, S Brueck, in Proc. Int. Symp. on Wirel. Commun. Systems, Application of coordinated beam selection in heterogeneous LTE-Advanced networks. (Aachen, Germany, 2011, pp. 730-734

19. D López-Pérez, I Güvenc, G de la Roche, M Kountouris, TQS Quek, J Zhang, Enhanced intercell interference coordination challenges in heterogeneous networks. IEEE Wirel. Commun. 18(3), 22-30 (2011)

20. IF Akyildiz, DM Gutierrez-Estevez, EC Reyes, The evolution to 4G cellular systems: LTE-Advanced. Phys. Commun. 3(4), 217-244 (2010)

21. 3GPP: Evolved universal terrestrial radio access (E-UTRA); physical channels and modulation (Rel. 10). 3GPP technical specification, TS 36.211, Ver. 10.3 .0 (2011)

22. 3GPP: Evolved universal terrestrial radio access (E-UTRA); physical layer procedures (Rel. 10). 3GPP technical specification, TS 36.213, Ver. 10.3.0 (2011)

23. J Hämäläinen, R Wichman, in Proc. IEEE Int. Symp. on Personal, Indoor and Mobile Radio Commun., Performance analysis of closed-loop transmit diversity in the presence of feedback errors. vol. 5 (Lisbon, Portugal, 2002), pp. 2297-2301

24. 3GPP: Physical channels and mapping of transport channels onto physical channels (FDD) (Rel. 7). 3GPP technical specification, TS 25.211, Ver. 7.8.0 (2002)

25. A Hottinen, O Tirkkonen, R Wichman, Multi-Antenna Transceiver Techniques for $3 G$ and Beyond. 1st edn. (John Wiley \& Sons, New York, 2003)

26. AA Dowhuszko, M Husso, J Li, J Hämäläinen, Z Zheng, in Proc. Future Network and Mobile Summit (Workshop on Broadband Femtocell Networks), Performance of practical transmit beamforming methods for interference suppression in closed-access femtocells. (Warsaw, Poland, 2011), pp. 1-12

27. A Papoulis, Probability, Random Variables, and Stochastic Processes. 3rd edn. (McGraw-Hill International Editions, 1991)

28. AA Dowhuszko, G Corral-Briones, J Hämäläinen, R Wichman, On throughput-fairness tradeoff in virtual MIMO systems with limited feedback. EURASIP J. Wirel. Commun. Network. 2009, 1-17 (2009)

29. J Hämäläinen, R Wichman, AA Dowhuszko, G Corral-Briones, Capacity of generalized UTRA FDD closed-loop transmit diversity modes. Wirel. Personal Commun. 54(3), 467-484 (2010)

30. AA Dowhuszko, G Corral-Briones, J Hämäläinen, R Wichman, in Proc. IEEE Int. Conf. on Commun, Achievable sum-rate analysis of practical multiuser scheduling schemes with limited feedback. (Glasgow, Scotland, 2007), pp. 4381-4386

doi:10.1186/1687-1499-2012-293

Cite this article as: Dowhuszko et al: Combined transmit beamforming and channel-aware scheduling for interference mitigation in femtocells. EURASIP Journal on Wireless Communications and

Networking 2012 2012:293.

\section{Submit your manuscript to a SpringerOpen ${ }^{\circ}$ journal and benefit from:}

- Convenient online submission

- Rigorous peer review

- Immediate publication on acceptance

- Open access: articles freely available online

- High visibility within the field

- Retaining the copyright to your article

Submit your next manuscript at $\boldsymbol{\triangleright}$ springeropen.com 